\title{
Origin and compensation of imaging artefacts in localization-based super-resolution microscopy
}

\author{
M. Erdélyi ${ }^{1}{ }^{*}, J$. Sinkó $^{1}$, R. Kákonyi ${ }^{1}$, A. Kelemen ${ }^{2}$, E. Rees $^{3}$, D. $\operatorname{Varga}^{1}$ and G. Szabó $^{1}$ \\ ${ }^{1}$ Department of Optics and Quantum Electronics, University of Szeged, Szeged, Dóm tér 9, 6720 Hungary \\ ${ }^{2}$ Department of Applied Informatics, University of Szeged, Boldogasszony sgt. 6, \\ 6725 Hungary \\ ${ }^{3}$ Department of Chemical Engineering and Biotechnology, University of Cambridge, New Museums Site, \\ Pembroke Street, Cambridge CB2 3RA, UK
}

\begin{abstract}
Interpretation of high resolution images provided by localization-based microscopy techniques is a challenge due to imaging artefacts that can be categorized by their origin. They can be introduced by the optical system, by the studied sample or by the applied algorithms. Some artefacts can be eliminated via precise calibration procedures, others can be reduced only below a certain value. Images studied both theoretically and experimentally are qualified either by pattern specific metrics or by a more general metric based on fluorescence correlation spectroscopy.
\end{abstract}

*Corresponding author: erdelyim@physx.u-szeged.hu

Keywords: Super-resolution microscopy, localization microscopy, imaging artefacts, localization error

\section{Introduction}

Since the first appearance of optical microscopes, visualization of small biological organelles has been one of the major fields of applications [1]. However, the development of microscopes with high magnification requires superior optical components, since the useful magnification is limited by the spatial resolution. Magnification above this practical limit, referred to as "empty magnification" [2], typically degrades image quality. In 1873 Ernst Abbe formulated the spatial resolution limit of a diffraction limited aberration-free imaging system: $\mathrm{R}=0.5 \lambda / \mathrm{NA}$, where $\lambda$ is the wavelength and NA is the numerical aperture [3]. Abbe also pioneered in the development of high quality microscope objectives (apochromats etc.) necessary for state-of-the-art microscope systems.

The spatial resolution in the visible region - widely used in bio- and medical research - is approximately $250 \mathrm{~nm}$, precluding the study of fine structures at a single molecule level. Imaging of cellular structures in the sub-diffraction area was considered impossible due to the above Abbe limit. However, in the last decades several super-resolution methods have been demonstrated [4], such as stimulated emission depletion (STED)[5, 6], structured illumination (SI) [7, 8] and localization-based (PALM, STORM, dSTORM etc.) [9, 10, 11, 12, 13] microscopes. All these techniques provide high spatial resolution but have their own limitations that constrain their applicability. In the localization-based techniques the fluorescent molecules are detected individually. The diffraction limited spots of individual molecules are fitted by the point spread function (PSF) [14] of the optical system. Usually, the PSF of the optical system is approximated by a Gaussian distribution, which facilitates fast and simple fitting algorithms with minor loss of accuracy. In localization-based microscopy the central position of the fitted curve is important because it corresponds to the position of the molecule. It is worth noting that the profile of the PSF at high NA, assuming quickly rotating fluorescent molecules, is very close to the Gaussian distribution $[\mathbf{1 5}, \mathbf{1 6}]$. Determination of the centre of the PSF is an order of magnitude more precise than the FWHM of the PSF, hence localization-based techniques can achieve a spatial resolution of $20 \mathrm{~nm}$ routinely [17].

However, all the proposed super-resolution systems including the localization-based methods are implemented into traditional diffraction-limited microscope systems. The physical parameters (optical 
aberrations, drift, stability etc.) of such systems were optimized for traditional imaging and not for superresolution. Therefore, system development, data acquisition and final image implementation require special attention [18]. In this paper we review some important errors and artefacts that can arise in localization based super resolution microscopy, and give methods for their minimization, and metrics to indicate whether they will cause significant problems. Such artefacts can introduce random or systematic localization errors, which lead to blurred or distorted super-resolved images. Elimination and minimization of such undesirable effects depend on the nature of the artefact. In this paper artefacts are ranked by their origin and will be classified as artefacts introduced by the system, the sample or the algorithms, respectively. The magnitude and distortion effects of the artefacts depend on the specific optical system, the studied sample and the applied localization algorithms. Localization based techniques require intensive image processing therefore artefacts introduced by different algorithms are more critical than in STED, where system artefacts arising from the complex imaging setup are more critical.

\section{Materials and Methods}

The critical optical components of the microscope system were simulated by OSLO, an Optics Software for Layout and Optimization [19]. The PSFs were calculated based on a polarization dependent ray tracing algorithm. The simplified imaging system consisted of a modelled high numerical aperture microscope objective (NA 1.41 and magnification 100×) [20] and a perfect lens (tube lens) with a focal length of $180 \mathrm{~mm}$. The refractive index and thickness of the immersion oil were matched to the cover slide and the depth of the ROI, respectively. The point-like sources (fluorescent dye molecules) were placed in water medium used as a homogeneous and isotropic sample. Polychromatic PSF was calculated for each emitter by means of a direct integration method $[\mathbf{1 9}, \mathbf{2 1}]$. The wavelength ranges were set taking into consideration the transmission windows of the emission filters [22] and were matched to the absorption/emission spectra of the most common dyes that can be excited at $405 \mathrm{~nm}, 488 \mathrm{~nm}, 561 \mathrm{~nm}$ and $635 \mathrm{~nm}$, respectively. In the image plane the pixel size was typically chosen to be $16 \mu \mathrm{m}$, the same as applied during the experiments. It was assumed that the fluorescent emission was unpolarized (degree of polarization=0) and independent of the excitation conditions (fluorescence anisotropy=0). The pixelated images of single molecules (PSFs) and the Strehl-ratio were saved and stored for further evaluation.

Test data were generated by testSTORM, an open source sample generator program [23, 24]. TestSTORM generates image stacks simulating realistic STORM measurements of defined samples. Four families of patterns can be selected: the star, array, vesicles and line samples. The geometrical parameters (radius of the vesicles, length of arms etc.) of the selected patterns, the photochemical parameters (characteristic life times, emission wavelength etc.) of the applied dye molecules, and acquisition parameters (frame rate, exposure time etc.) of the optical system can be defined. The 3D PSF of the individual, fast-rotating dye molecules calculated by OSLO was implemented and used for physically accurate simulation.

There are several algorithms proposed for localizing individual blinking molecules [25, 26]. In this paper the simulated image stacks were evaluated and visualized by rainSTORM, an open source code for MatLab[27, 28]. In addition to basic features rainSTORM provides methods for fiducial marker tracking and drift correction, basic image simulation for test benching and validation; batch processing of multiple files; chromatic offset correction; and molecular trajectory imaging. The output data provided by testSTORM can be used as highly-realistic data for rainSTORM.

dSTORM super-resolution images were captured using a modified Olympus IX71 inverted, objective based total internal reflection (TIRF) microscope platform. Fluorescence and excitation lights were spectrally separated by a full multi-edge filter set (LF405/488/561/635-A-000 from Semrock). A diode laser operating at $640 \mathrm{~nm}$ (iBeam smart, $150 \mathrm{~mW}$ from Toptica Photonic AG, Graefelfing, Germany) was fibre coupled, expanded and focused at the edge of the back focal plane of the objective (UAPON 100XOTIRF, NA=1.49). The perfect illumination condition of the sample was controlled by the fine positioning of the focused beam. Under TIRF illumination only the evanescent field with a penetration depth of about $150 \mathrm{~nm}$ excited the sample and reduced the background fluorescence significantly. Highly inclined illumination was also used in some 
cases to improve the signal to noise ratio. The beam size of the excitation laser was matched to the FOV of the imaging system limited by the low-noise, highly sensitive electron-multiplying EMCCD camera (Andor iXon 897). The overall magnification of the microscope was 100. Multicoloured fluorescent beads (100 $\mathrm{nm}$, Invitrogen Molecular Probes, T7279) were used to study the chromatic and mechanical drifts of the system and the samples, respectively.

\section{Results and Discussion}

\subsection{System artefacts}

The localization precision - the error of determining the measured position of the fluorescent molecule strongly depends on the number of photons collected by the optical system and the noise.[29, 30] Thompson showed that the pixel size of the CCD camera also affects the localization precision[31], and the ideal ratio of the pixel size $(a)$ and standard deviation $(s)$ of the point-spread function (PSF) of the imaging system is at around $a / s=1$. It means that photons emitted by a single molecule are captured typically by a $5 \times 5$ CCD pixel area. Due to this sampling density, small deformations of the PSF introduced by optical aberrations barely affect real measurements. [15]

\subsubsection{Monochromatic aberrations}

Monochromatic aberrations such as spherical aberration, coma and astigmatism can distort the PSF of the optical system especially when the source is located in an off-axis position. In localization microscopy the imaged FOV is typically small ( $<50$ microns) and the selected ROI is centred to the optical axis. However, in some special cases the FOV can be significantly larger (single molecule tracking) and the ROI can be at an offaxis position (imaging of different regions without stage translation), when the PSF of molecules located further from the optical axis can be distorted. The question is how monochromatic aberrations affect the localization accuracy? Simulations of a real microscope system via OSLO were performed to answer this question.

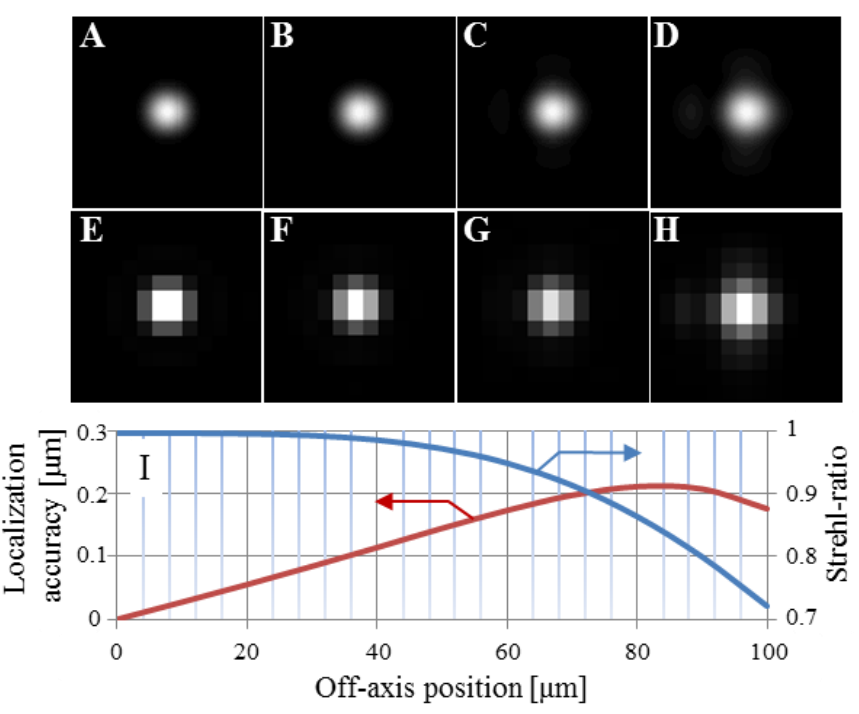

Figure 1. High resolution (A-D) and under-sampled (E-H) calculated images of isolated point-like sources on the axis $(A, E), 50$ microns off-axis $(B, F), 84$ microns off-axis $(C, G)$ and 100 microns off-axis $(D, H)$ positions. (I) The localization error increases with off-axis position to a maximum at 84 microns.

Figure 1 depicts the calculated high resolution (Fig 1. A-D) and pixelated (Fig 1. E-H) point-spread functions. The high resolution images show no distortion when the off-axis position is smaller than 50 microns. Images of point-like sources located further than 50 microns from the optical axis have asymmetric slide lobes. The Strehl 
ratio reveals the aberrations in a more sensitive way. In contrast, the visual evaluation of the under-sampled images (as captured by a CCD) do not show significant spatial dependence. With increasing off-axis position, the PSF spreads out asymmetrically, which can be hardly visualized by eye: but when the PSF is computationally fitted by a localization algorithm, the centre position of the fitted Gaussian curve is displaced from the position of the fluorescent molecule, which distorts the super-resolution image. Figure 1 I shows the localization accuracy (the distance of the exact and localized position of the molecules) as a function of the offaxis position. Below 50 microns the accuracy shows a linear dependence: the localized position is always closer to the optical axis than the exact position of the molecule. Above 50 microns the second order term dominates and accuracy has an extremum of $200 \mathrm{~nm}$ offset at the 82 micron off-axis position. This result proves that microscope systems are optimized for diffraction limited imaging: the error introduced by monochromatic aberration at the edge of a relatively large (200 micron) FOV is still smaller than the diffraction limit $(250 \mathrm{~nm})$. However, in localization microscopy such a small inaccuracy may be significant and could lead to the misinterpretation of the final image. For example, in imaging of extended highly structured surfaces, which was proposed to provide nanoscopic rulers for the calibration of super-resolution microscopy.[32, 33] However, such a small inaccuracy in single colour LOC imaging of soft biological samples is basically insignificant. Localization inaccuracy of $200 \mathrm{~nm}$ typically cannot be visualized and does not lead to misinterpretation of a single colour image since biological samples are typically not highly structured.

\subsubsection{Chromatic offset}

Multicolour localization microscopy requires multiple labelling and sequential [34] or simultaneous [35] excitation and localization of fluorescent molecules. In case of simultaneous excitation a dichroic mirror is applied to separate fluorescent light emitted by different dyes. After the spectral separation dual or singledetector [36] configurations are used to acquire images. Image registration is an essential task due to the slight optical difference between the two channels.[37] Multicoloured beads are the most widely used fiducial markers in localization microscopy, because of their stability and relatively small size [18, 38]. In case of sequential excitation, the different fluorescent dyes are excited separately in time and the full chip size of a single detector can be used. However, registration of the two images is still necessary because of the lateral chromatic aberration of the imaging system [39]. Such registration can be done with a single bead scanned through the FOV or multiple beads located randomly or in an ordered way. Captured two-colour images of white fluorescent beads at different positions of the FOV can be seen in Fig. 2 before and after image registration.
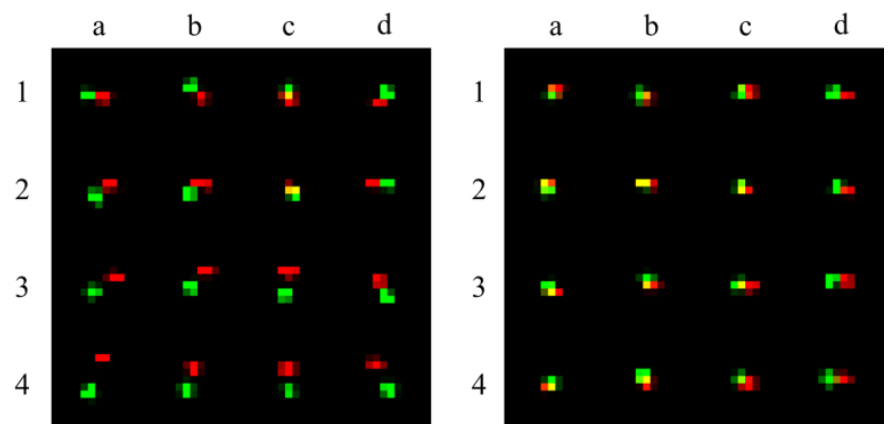

Figure 2. The super-resolution image of a 40x40 micron FOV was divided into 16 regions. A white fluorescent bead at the centre of each region is shown before (left) and after (right) optical offset correction. A zoomed-in scale of $16 \mathrm{~nm}$ per pixel is used for this reconstruction, with the empty space of 10 microns between the beads cropped out. Before the correction the cylindrical symmetry of optical offset can be seen clearly (probably with a centre in the $2 \mathrm{C}$ region). A single translation cannot improve the two colour image: only spatially-dependent optical offset correction can correct the chromatic distortion.

\subsubsection{D drift (thermal and mechanical):}


The detection of single fluorescence molecules requires a stable optical system with thermal and mechanical drifts smaller than the localization precision $(\approx 10 \mathrm{~nm})$ throughout the entire data acquisition time so that the visualised fluorophore density map does not suffer from "motion blur". In practice one captures data typically over 2-3 minute intervals, but for complex samples the acquisition process may take tens of minutes or more, depending on factors such as the field of view $(F O V)$, labelling density and the detection efficiency. Mechanical and thermal stability of the system is critical, since image drift (which is either random or shows a trend) reduces the final image contrast. Optical components are aligned as close to the surface of the optical table as possible to promote mechanical stability. The table is usually air-floated and the optical setup is protected by an enclosure box to reduce movement due to thermal and mechanical fluctuations. Air-shielding is recommended to minimize thermal drift and vibration. The camera, usually a high sensitivity EMCCD, is often attached directly to the microscope frame and heats upon being switched on. It may take up to 5 hours for the system to equilibrate after being switched on and to reach optimal stability. In practice one should always check that the required localization precision has been achieved, before experiments are conducted. In critical situations active or passive control measures may be required to minimize drift. [40]. State-of-the-art microscope frames are equipped with autofocus systems typically based on the displacement measurement of the back-reflected IR beam from the coverslip/sample interface. Previous generation frames can also be equipped with autofocus systems mounted onto one of the microscope ports. Alternatively, a nosepiece stage (e.g., IX2-NPS, Olympus) can be used to minimize the drift of the specimen relative to the objective. [13] These solutions can typically keep the sample in focus with a precision of $20 \mathrm{~nm}$.

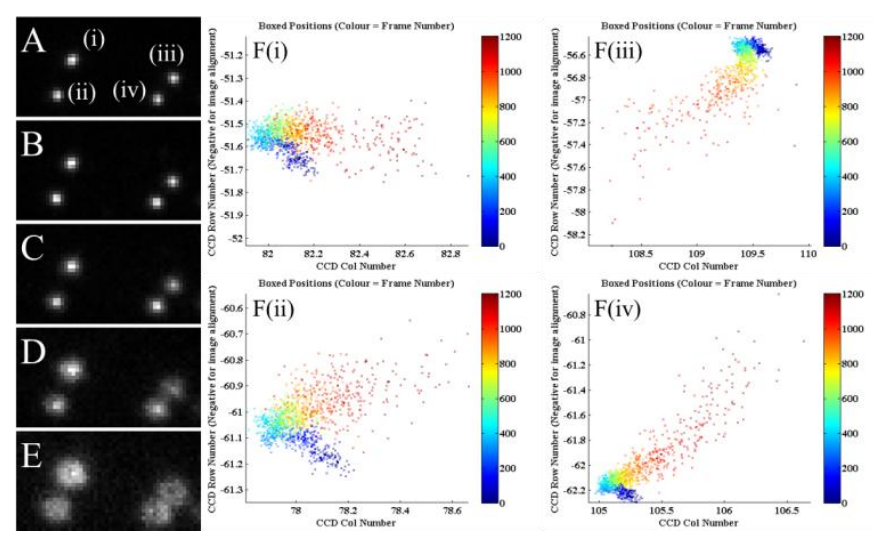

Figure 3. Localization of four (i-iv) fluorescent beads in the focus (A) and at different defocused positions (B-

E). Time lapse localization of the individual beads (F) reveals the asymmetry of the PSF and reduced localization precision.

Fiducial markers such as fluorescent beads can be used for drift correction, too. Figure 3 depicts the images of four fluorescent beads in (A) and out of (B-E) the focus position. As many as 1200 image frames were captured in sequence when the focus lock was off. In case of $\mathrm{E}$, a ring shape of the captured patterns shows that the whole focus drift during the data acquisition was approximately equal to the depth-of-field (600 nm). Visually the defocused patterns have perfect cylindrical symmetry. However, time lapse localization of the individual beads (Figure 3F) reveals the asymmetry of the under-sampled PSFs. At the beginning (blue-green dots) the localization error was in the range of 0.1 pixel. At around the 700th frame (yellow dots) the defocus started to change more drastically. The increased scattering of the red dots (localizations of beads in frames between 9001200) proves the asymmetry of the defocused PSF. This experiment shows that fluorescent beads can be applied as fiducial markers only if they are exactly in the same image plane as the sample.

\subsection{Distortions introduced by the sample}


The sample itself affects the imaging process, especially when a deeper region in an extended sample is imaged. If a thick sample is inspected, the sample part between the focal plane and the cover glass affects the imaging and causes distortions in the PSF, which can lead to image deterioration. The out of focus noise, which is caused by the fluorescence of the out of focal regions of the sample, also degrades the quality of the final image. This can be minimized by means of inclined illuminations.

\subsubsection{Distortion of multicolour molecule images located at a deeper region in the sample.}

The region located between the focal plane and the surface of the cover plate can affect the imaging in both the single and multicolour imaging $[\mathbf{3 8}, \mathbf{4 1}]$.

To study the deeper region imaging, four modelled vesicles were imaged at several depths in the sample in two colours. The position of the dye molecules were generated by means of the testSTORM [23, 24] test pattern generator software. The PSFs of the molecules were calculated with the OSLO optical design software. RainSTORM localization software [28] was used for localization and image reconstruction. The diameter of the vesicles was chosen to be $75 \mathrm{~nm}$, and 400 molecules were linked to each vesicle. The centres of the vesicles were placed in the focal plane on a frame sized $256 \times 256 \mathrm{CCD}$ pixels $(1 \mathrm{CCD}$ pixel equals $160 \mathrm{~nm}$ in the focal plane) (Fig. 4. A). Two cases were analysed when the focal plane was at a depth of $80 \mathrm{~nm}$ (Fig. 4. B) and $5 \mu \mathrm{m}$ (Fig. 4. C) from the cover plate, respectively. The lateral distance between the vesicle centres was 45 CCD pixels. 300 frames were captured in each case. The sample medium was assumed to be water and homogenous. The applied wavelength windows for the OSLO polychromatic PSF calculation were: 663-690 nm (red) and 502-544 $\mathrm{nm}$ (green) according to the transmission windows of a commercially available emission filter (LF405/488/561/635-A-000 from Semrock). At a larger lateral distance from the optical axis (from vesicle position 1 to vesicle position 4) the two colour-channels (red and green) became more divergent due to the chromatic aberrations and the chromatic offset increased. The white cross marks the position of the vesicle centres in case of ideal imaging. The difference between the $80 \mathrm{~nm}$ and $5 \mu \mathrm{m}$ was found insignificant in a homogenous medium such as cells cultured on a coverslip. However, in thicker samples such as brain tissues, the inhomogeneities within the sample can cause relevant discrepancies: blurred structures, decreased localization precision or high number of mislocalization. The type of the error highly depends on the sample structure. The correction or elimination of this error is a challenging field in the microscopy. The application of inclined illuminations can cure or mitigate this problem (see section 3.2.2).
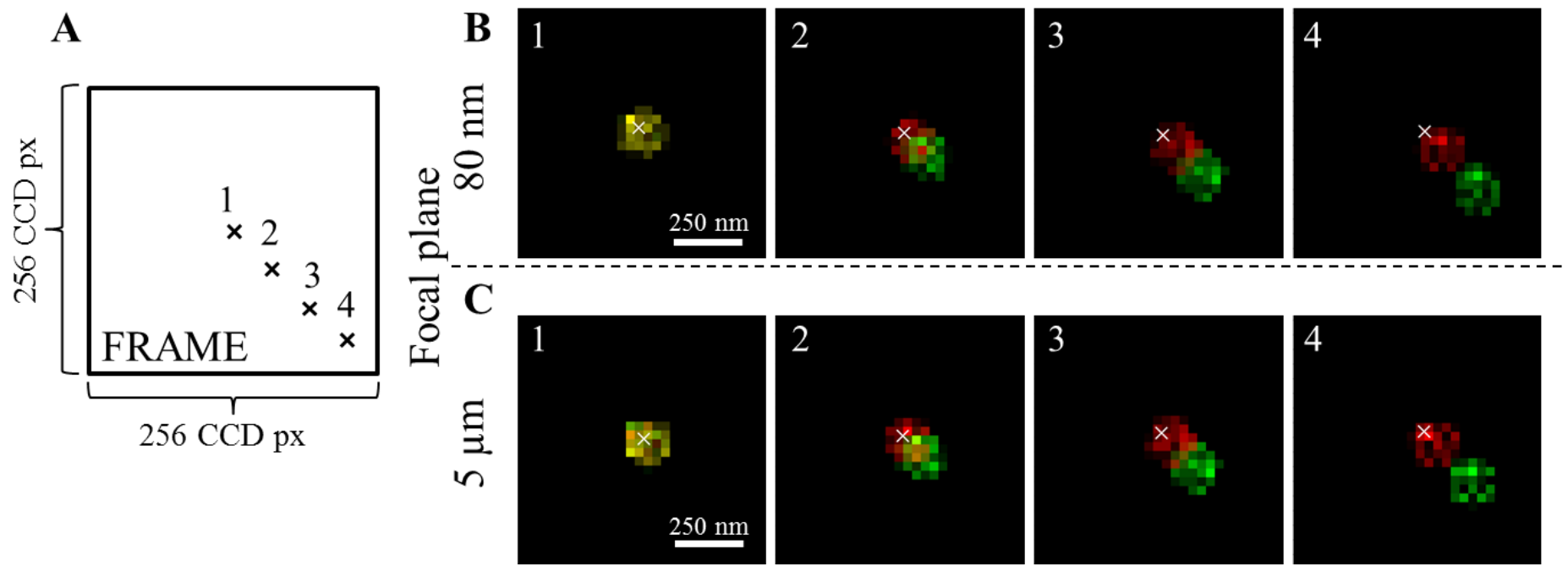

Figure 4. The effect of deeper sample imaging, (A) the centre position of four vesicles on the frame (sized $256 \times 256$ CCD pixels, 1 CCD pixel equals $160 \mathrm{~nm}$ in the focal plane), (B) and (C) super-resolved pictures of the four vesicles in case of $80 \mathrm{~nm}$ and $5 \mu \mathrm{m}$ deep imaging, respectively. 
The chromatic offset was also studied in the case of extended samples, where the defocus became significant during imaging within the Depth Of Field (DOF). The degree of the offset depends on the amount of defocus. In the simulations the focal plane was at a distance of $5 \mu \mathrm{m}$ from the cover plate. At this depth the aberrations become more significant especially in case of oil-objectives. $[\mathbf{4 2}, \mathbf{4 3}]$ The range where the analysis was carried out was $+/-300 \mathrm{~nm}$ around the focal plane in $\mathrm{z}$ direction, according to the typical size of the DOF. The method was the following: emitters were placed in a matrix form on the frame at several planes in $\mathrm{z}$ direction (at $300 \mathrm{~nm}, 0 \mathrm{~nm}$ and $300 \mathrm{~nm}$ ). The size of the frame was $256 \times 256$ CCD pixels. 1 CCD pixel was $160 \mathrm{~nm}$ in the focal plane. The polychromatic PSFs of the emitters were calculated in OSLO in two emission wavelength windows (663-690 $\mathrm{nm}$ and 502-544 $\mathrm{nm})$. The PSFs were localized with rainSTORM, and the lateral dependence of chromatic offset (difference between the two channels) was calculated in all planes. The offset was found to be cylindrically symmetric relative to the optical axis in the lateral direction in each $z$ plane. Cross-sections were depicted for each plane (Fig. 5). The figure shows, that on the edge of the FOV the value of the chromatic offset is greater than one CCD pixel $(160 \mathrm{~nm})$. Furthermore it is not symmetric relative to the focal plane in $z$ direction. The difference between the offset in the $-300 \mathrm{~nm}$ and $300 \mathrm{~nm}$ planes is 0.75 CCD pixel $(120 \mathrm{~nm})$, therefore different calibration functions are required in the different $z$ planes.

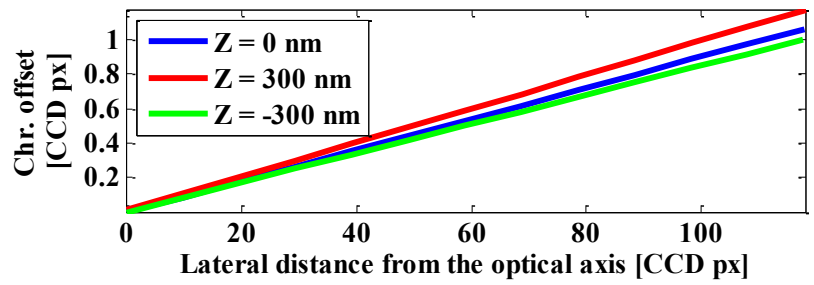

Figure 5.The chromatic offset at several planes in the sample. The focal plane was placed $5 \mu \mathrm{m}$ far from the cover plate. The full size of the frame was $256 \times 256$ CCD pixels. 1 CCD pixel was $160 \mathrm{~nm}$ in the focal plane.

\subsubsection{Fluorescent background reduction via inclined illuminations}

The fluorescent background caused by out of focus fluorescence degrades the final image quality especially in the localization-based microscopy technique, where the precision of the localization highly depends on the background level [31]. Therefore, new illumination methods were developed for the out of focus signal reduction instead of using the conventional epifluorescence illumination (EPI). When the region of interest is close to the cover plate (typically 50-100 nm) TIRF illumination is preferred [44], e.g. by lateral beam translation in the back-focal plane (Fig. 6. A). If the region of interest is $1 \mu \mathrm{m}$ far from the cover plate, VAEM (Variable Angle Epifluorescence Microscopy) can be applied [45], where the focus of the illumination beam is moved into an off-axis position in the back focal plane (Fig. 6. B). In case of deeper plane imaging $(\sim 5 \mu \mathrm{m}$ from the cover plate), the fluorescent background can be reduced via HILO illumination (Highly Inclined and Laminated Optical sheet microscopy) [46]. In this case the incoming beam must be both shifted and inclined in the back-focal plane (Fig. 6. C) to keep the illumination of ROI centred.

A

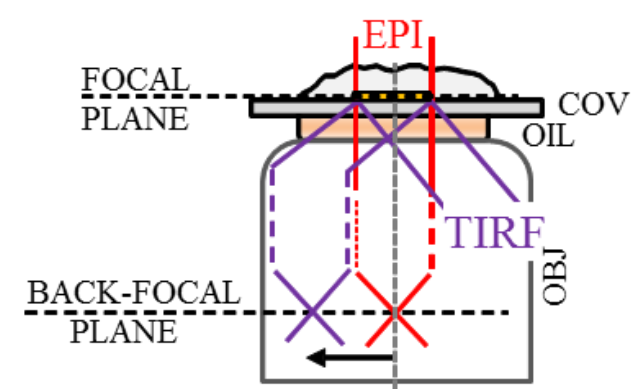

$\mathrm{B}$

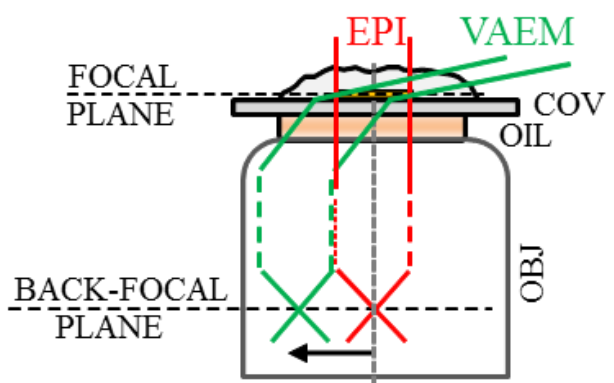

$\mathrm{C}$

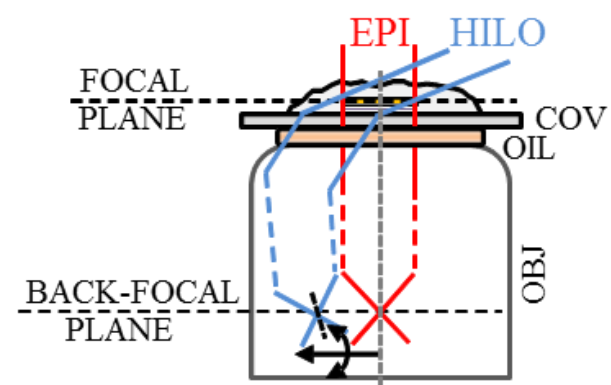

Figure 6. Suggested inclined wide-field illuminations with the focal plane located at (A) 0.05 , (B) 1 and (C) 5 $\mu \mathrm{m}$ deep in the sample. The conventional epifluorescence (EPI) illumination is also depicted in all cases. 
The required inclination and translation can be introduced into the illumination path by means of $4 \mathrm{f}$ systems and rotatable mirror mounts placed in the conjugate planes or movable lens mounts. In case of the firstly mentioned alignment ( $4 \mathrm{f}$ systems), two $4 \mathrm{f}$ systems are required with rotatable mirrors in the conjugate planes of the focal plane and the back-focal plane, respectively, see Fig. 7. A. In the second case (movable lens mounts), the tube lens is placed in a translational movable mount, which introduces the inclination in the back-focal plane. To introduce the inclination in the focal plane, the incoming beam must be translated too (e. g. by means of a rotatable plane parallel plate [18]), see Fig. 7. B. The 4f systems require more optical elements, which can lead to unacceptable intensity loss, however, these systems are quite stable and their feasibility is high.

A

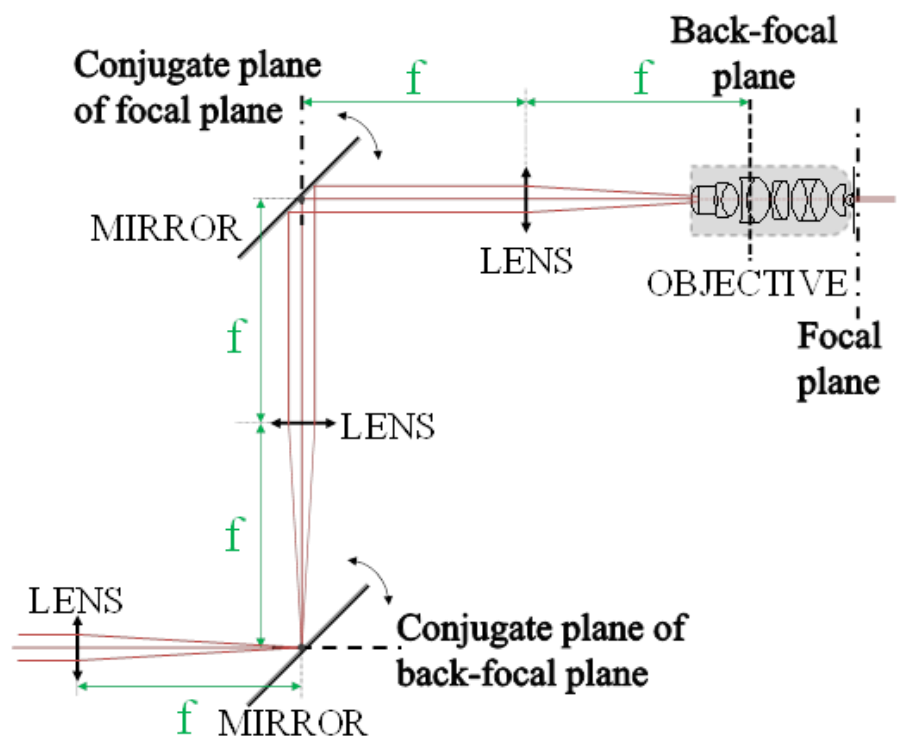

B

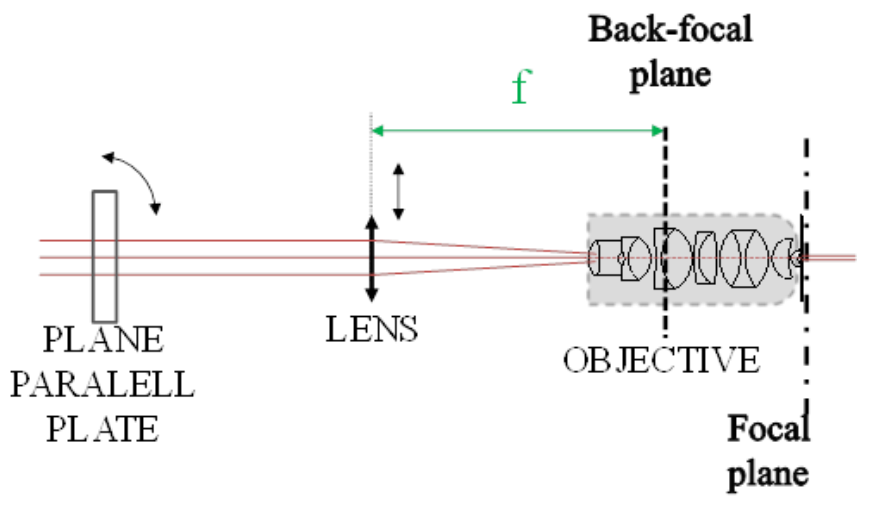

Figure 7. The realisation of inclined illuminations by means of (A) rotatable mirror mounts and 4f systems and (B) movable lens mounts

A detailed study of inclined wide-field illumination can be found in [47] with enhanced consideration of issues apparent in the localization-based microscopy techniques.

\subsection{Artefacts introduced by the algorithms}

The software methods used to infer fluorophore positions and reconstruct the super-resolved image can also be a source of artefacts. The presence of different artefacts strongly depends on how accurately the localization algorithm handles a particular set of experimental data. One key requirement for successful localization microscopy is that fluorophore positions are determined precisely, but even more important is that false positive localizations are excluded. Both these properties require raw fluorescence image data which is sufficiently sparse for point-spread-function fitting algorithms to operate effectively. In a localization microscope system it may be possible to obtain sparse fluorophore images by optimizing the experimental parameters: the exposure time, dye parameters, labelling density and sample geometry. In this section we show how simulations can be used to identify suitable parameters. We also present metrics of image quality that can indicate whether the super-resolution image produced from a given set of raw data is accurate.

When using a single molecule fitting algorithm for localization microscopy, it is essential to avoid situations where two or more molecules produce overlapping point-spread functions (PSFs). Otherwise a single spurious localization may be identified between two or more real fluorophore positions, which leads to several types of artefact in the reconstructed image. The probability of recording overlapping PSF spots increases with the duty cycle of the dye, the labelling density of the sample and the exposure time of the camera. The probability of 
overlapping is also higher if the geometry of the sample is highly detailed: one-dimensional, linear actin filaments tend to have few fluorescent labels, even if they are closely spaced, and are more amenable to localization microscopy than extensive 3D objects. The probability of overlapping PSFs can be decreased by shortening the camera exposure time, but this may decrease the number of collected photons and degrade resolution.

Experimental optimization methods are expensive and time consuming, and simulations can speed up the optimization stage of localization microscopy. In the following section, image simulations generated for defined specimen geometry by the testSTORM software will be used to identify the amount of artefacts arising from a localization microscopy experiment. There are many different applications of localization microscopy, and the geometries of specimens studied in different applications can vary widely. Important specimen geometries that can be simulated using testSTORM include: spherical vesicles, crossed lines, 3D spirals [48], and small structures with n-fold rotational symmetry such as nuclear pore complexes [49]. To demonstrate the usage of metrics to quantify image data quality, test geometries based on simulated vesicles and crossed lines objects are presented. For these known samples, objective measures of image quality can be defined. Such specimen-dependent metrics are useful because they can be used to validate sample-independent metrics of image quality. A sample-independent metric of image quality based on fluorescence correlation spectroscopy (FCS) is then given to evaluate whether experimental data should produce a super-resolved image free from artefacts. The emphasis of this section is determining whether the localisation algorithm produces artefacts due to fluorophore density, and so single colour simulations are presented, excluding the optical artefacts presented above. 


\subsubsection{Bridge artefacts in vesicular samples}

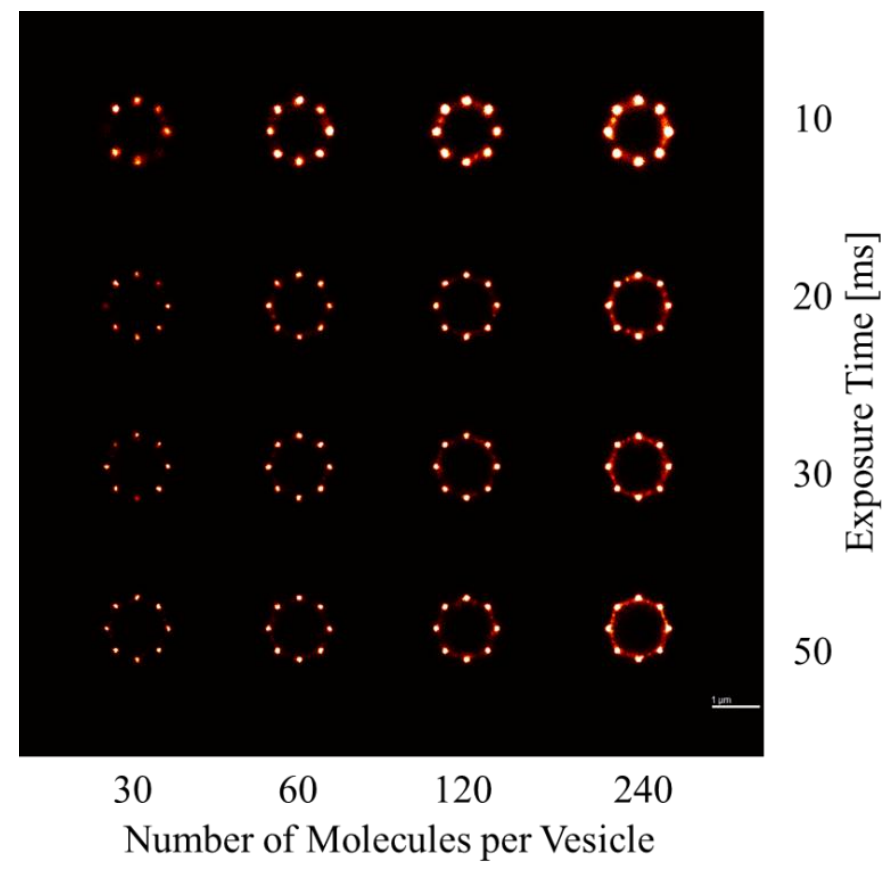

Contrast
\begin{tabular}{|l|l|l|l|}
\hline 0,81 & 0,88 & 0,87 & 0,86 \\
\hline 0,92 & 0,91 & 0,91 & 0,85 \\
\hline 0,91 & 0,94 & 0,90 & 0,86 \\
\hline 0,93 & 0,93 & 0,90 & 0,84 \\
\hline
\end{tabular}

Bridge localizations

Good localizations

\begin{tabular}{|l|l|l|l|}
\hline 38.8 & 115 & 184 & 292 \\
\hline 63.8 & 134 & 219 & 365 \\
\hline 68.3 & 160 & 232 & 391 \\
\hline 98.5 & 192 & 292 & 425 \\
\hline
\end{tabular}

\begin{tabular}{|l|l|l|l|}
\hline 6.13 & 12.9 & 21.6 & 39.1 \\
\hline 4.63 & 11.6 & 19.6 & 51.5 \\
\hline 5.25 & 8.25 & 22.4 & 52.4 \\
\hline 6.25 & 12.8 & 28.3 & 67.6 \\
\hline
\end{tabular}

FCS number density metric

\begin{tabular}{|l|l|l|l|}
\hline 0.03 & 0.13 & 0.22 & 0.52 \\
\hline 0.06 & 0.16 & 0.29 & 0.63 \\
\hline 0.07 & 0.16 & 0.29 & 0.6 \\
\hline 0.08 & 0.2 & 0.37 & 0.61 \\
\hline
\end{tabular}

Figure 8. Super resolved images reconstructed from image data simulated for eight vesicles arranged on a circle of $640 \mathrm{~nm}$ radius. The tabulated image quality metrics are: the Contrast, or proportion of localisations corresponding to real fluorophores; the total number of Good localisations which arise from a single fluorophore; the total number of spurious localisations defined as Bridge localisations due to overlapping fluorophore images in the raw data; and the FCS number density metric which estimates the average number of potentially-overlapping fluorophore images that contribute to the localisations (Section 3.4.2). Provided the

FCS number density metric is much less than one, it is likely that few bridge localisations are detected.

Image sequences were generated by means of testSTORM using dye parameters typically achieved with Alexa Fluor $647[\mathbf{2 4 , 5 0 ]}$. These parameters are: on state lifetime: $50 \mathrm{~ms}$, duty cycle: 0.0005 , number of frames: 2000, the expected number of collected photons per on cycle: 3823 . The exact diameter of the simulated vesicles is $60 \mathrm{~nm}$, and the fluorophore linker length is $8 \mathrm{~nm}$.

Fig. 8 shows the super-resolution images resulting from different labelling density and camera exposure times. At $50 \mathrm{~ms}$ exposures, the reconstruction of the vesicles loaded with 30 and 60 molecules / vesicle are well separated and distinguishable. On increasing the labelling density, spurious localizations appear between the vesicles and seemingly imply the presence either of extra vesicles (at 120 molecules / vesicle), or interconnections between the real ones (here, at 240 molecules / vesicle). We call this fake interconnect between the real features a bridge artefact. Decreasing the exposure time might seem to be a good strategy to decrease the occurrence of the overlapping PSF spots in the recorded image sequence. This does indeed 
mitigate the bridge artefacts, but with shorter exposure times the worse localization precision leads to blurring and an increase in the apparent size of the vesicles. The array of simulations enables experimental parameters to be optimised, because the specimen geometry is known and each fluorophore position determined by the localisation microscopy algorithm can be rated as good if it corresponds to a real fluorophore or as a bridge artefact if it lies between two fluorophores due to overlapping images. The contrast parameter defined as $\left(\mathrm{N}_{\text {good }}\right.$ $-\mathrm{N}_{\text {bridge }} / \mathrm{N}_{\text {good }}$ in Fig. 8. We found that a high contrast parameter correlates with our visual assessment of the best super-resolution image quality, for this simulated specimen sample. A high contrast is desirable, but evaluating this metric requires complete knowledge of the specimen which is unavailable in real experiments. However, metrics that can be measured from the raw image data without any knowledge of the sample can be validated against these known simulations, and hence the FCS number density metric described in Section 3.4 for estimating the density of photoswitching fluorescent molecules can be shown to correlate with the amount of bridge artefacts affecting the images of vesicular samples such as those in Fig. 8.

\subsubsection{Edge artefacts}

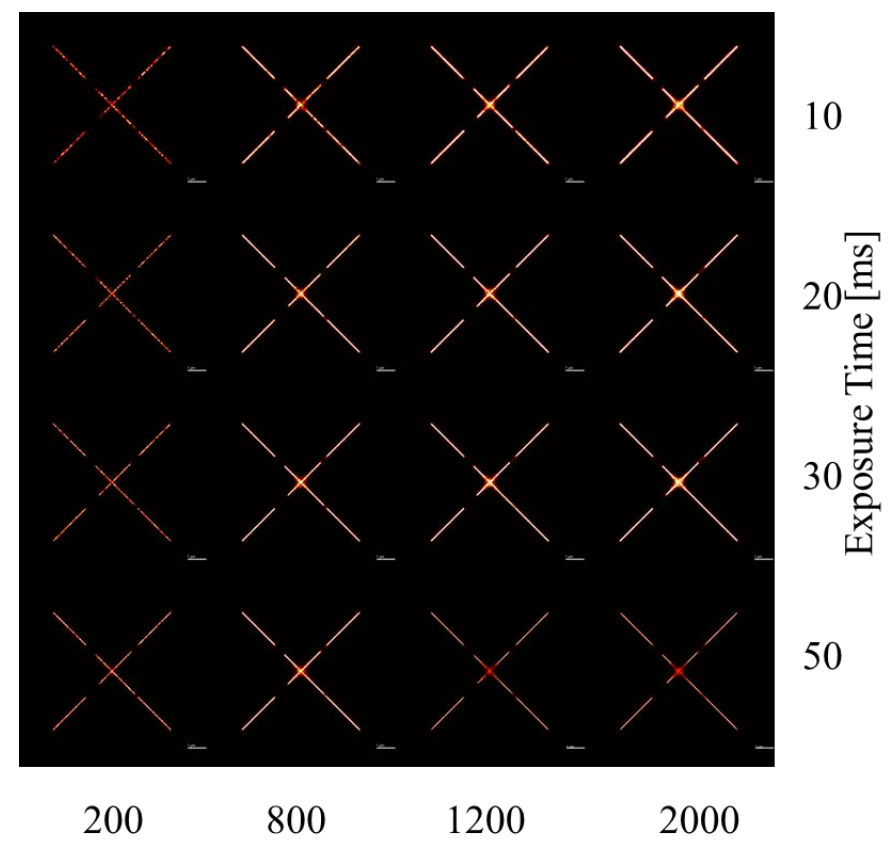

Labelling Density [Molecules / $\mu \mathrm{m}]$

\begin{tabular}{|c|c|c|c|}
\multicolumn{1}{c}{$\mathrm{C}_{\text {cross }}(0-500 \mathrm{~nm})$} \\
\hline 0.02 & 0.65 & 0.6 & 0.77 \\
\hline 0.18 & 0.51 & 0.68 & 0.70 \\
\hline-0.08 & 0.53 & 0.59 & 0.76 \\
\hline 0.14 & 0.65 & 0.63 & 0.81 \\
\hline
\end{tabular}

\begin{tabular}{|c|c|c|c|}
\multicolumn{3}{c}{$\mathrm{C}_{\text {Line }}(3000-3500 \mathrm{~nm})$} \\
\hline-0.2 & 0.18 & 0.16 & 0.16 \\
\hline-0.21 & -0.022 & 0.31 & 0.23 \\
\hline-0.64 & -0.042 & 0.28 & 0.17 \\
\hline 0.14 & 0.086 & 0.08 & 0.29 \\
\hline
\end{tabular}

$\mathrm{C}_{\text {Gap }}(1800-1950 \mathrm{~nm})$
\begin{tabular}{|c|c|c|c|}
\hline 0.89 & 0.88 & 0.86 & 0.71 \\
\hline 0.93 & 0.83 & 0.78 & 0.75 \\
\hline 0.93 & 0.89 & 0.69 & 0.69 \\
\hline 0.91 & 0.72 & 0.78 & 0.69 \\
\hline
\end{tabular}

FCS $(2250-4250 \mathrm{~nm})$
\begin{tabular}{|c|c|c|c|}
\hline 0.26 & 0.82 & 1.3 & 2.2 \\
\hline 0.25 & 1 & 1.4 & 2.4 \\
\hline 0.22 & 1 & 1.6 & 2.4 \\
\hline 0.31 & 1.1 & 1.6 & 2.8 \\
\hline
\end{tabular}

Figure 9. Super-resolved images of crossing lines with different sized gaps and tabulated metric data. For the definitions of the Cross, Gap and Line parts, see figure 11. The results for the $250 \mathrm{~nm}$ gap width case are shown. The tabulated FCS image quality metrics are defined in Section 3.4.2. 
In Fig. 9, image sequences were simulated for crossing-lines samples. Each arm of the cross specimen is defined to contain a gap (clockwise from bottom left arm, the gap is $1000 \mathrm{~nm}, 750 \mathrm{~nm}, 500 \mathrm{~nm}$, and $250 \mathrm{~nm}$ ). The fluorophore properties were set to the same typical values given for the vesicles case (Fig. 10), and the ability of a sparse localization microscopy algorithm to identify the gaps in the real specimen illustrated in Fig. 10 is tested.

In this situation, there is again an optimal labelling density that can be identified by visually assessing the super-resolution images produced by an array of simulations. At low labelling density (here, 200 molecules $/ \mu \mathrm{m}$ ) the brightness of the lines in the reconstructed image is inhomogenous and furthermore, since the labelling of the sample fails to satisfy the Nyquist sampling theorem in places, spurious extra gaps are implied. High labelling density does ensure that the reconstructed lines are of homogenous brightness, but the genuine gaps in the specimen become filled with false localizations, making the structure appear spuriously continuous. In this case, overlapping PSFs produce bridge artefacts which adversely affect the measurement of gaps, or the observation of edges, in otherwise continuous samples.

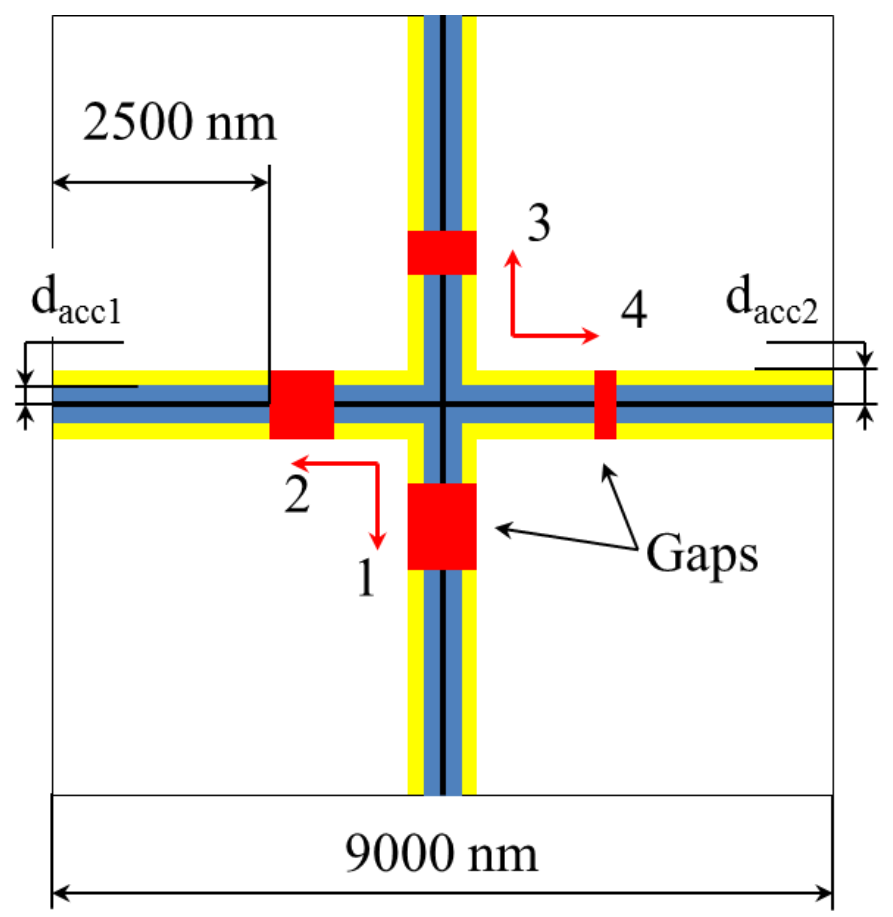

Figure 10.The schematic for evaluation of the crossing-line sample. The numbers (1-4) are indicating different gap sized $(1000 \mathrm{~nm}, 750 \mathrm{~nm}, 500 \mathrm{~nm}$, and $250 \mathrm{~nm})$ parts of the cross, see fig. 11.

Objective but sample-dependent metrics of super-resolution image quality for the crossed-lines samples were established as follows. The localizations were classified into subcategories based on their distances from the nearest line of the cross. Good localization distances are smaller than $\mathrm{d}_{\mathrm{acc} 1}$ and bad localization distances are between $d_{a c c 1}$ and $d_{a c c 2}$, see Fig 10 . The values of $d_{a c c 1}$ and $d_{a c c 2}$ parameters in this analysis were 25 and $160 \mathrm{~nm}$ respectively. Histograms were made from the localizations with $50 \mathrm{~nm}$ binning, the distance was measured from the lines crossing, see Fig 11. 

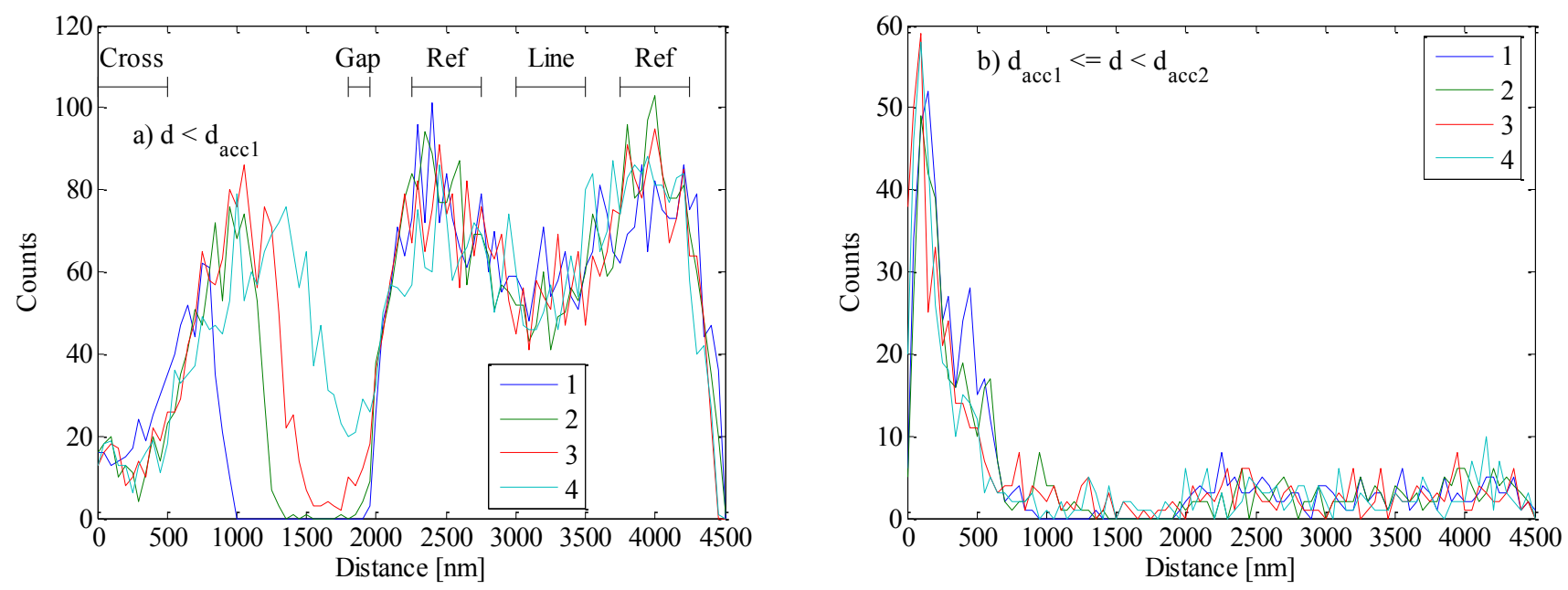

Figure 11. Distance histograms of good a) and bad b) localizations. The labelling density is 2000 molecules / $\mu \mathrm{m}$ and the exposure time is $50 \mathrm{~ms}$. Line parts used to calculate metrics: Cross, Gap and Line. The Ref line parts were used as common reference in contrast calculations.

Figure 11 shows the number of localizations as a function of distance from line crossing at 2000 molecules / $\mu \mathrm{m}$ labelling density and $50 \mathrm{~ms}$ exposure time. Three different artefacts could be observed: a) The number of good localizations decreases near the crossing of lines, while the number of bad localizations increases $(0-500 \mathrm{~nm})$. b) The gap between the line parts is filled with false localizations especially in the $250 \mathrm{~nm}$ gap width (1800-1950 nm). c) The line part with homogeneous labelling has decreased brightness in the central part (3000-3500 nm). Three contrast type metrics were defined to quantify these artefacts. The mean number of good localizations was determined in the corresponding sections of the lines. The central part, the gap and line part was assessed by means of averaging the number of good localizations in the $0-500 \mathrm{~nm}, 1800$ $1950 \mathrm{~nm}$ and the 3000-3500 $\mathrm{nm}$ distance ranges, respectively. The reference was the average number of localizations in the distance regions of $2250-2750 \mathrm{~nm}$ and $3750-4250 \mathrm{~nm}$. The calculated metric was the Weber contrast multiplied by minus one: $\left(\mathrm{N}_{\text {ref }}-\mathrm{N}\right) / \mathrm{N}_{\text {ref }}$. Note that the number of localizations in the reference area is typically higher than the analysed section of the lines.

The $\mathrm{C}_{\text {cross }}$ describes the brightness loss near the line crossing. Low values mean no artefact presence while high values mean the presence of bridge artefacts, which lead to an excess number of localisations between the lines of the cross. A second region of the sample is the centre of a line of the cross. Overlapping fluorophore images may lead to rejection of signals here, and the brightness loss of the centre of the homogenous line part is characterized by $\mathrm{Cline}_{\text {line }}$ The lower contrast value means the lack of artefacts: i.e. the centre of the line is the same brightness as its edges. There are negative values at 200 molecule / $\mu \mathrm{m}$ labelling density, because the labelling density is too low to satisfy the Nyquist criterion, the reconstructed image of the continuous line contains false gaps. Increasing the labelling density the contrast also increases indicating that the artefact is more influential, and above 800 molecules / $\mu \mathrm{m}$ labelling density this artefact is more and more apparent. A third region of the specimen in the gap in the arms of the cross: this region should contain zero localizations, and so $\mathrm{C}_{\text {gap }}$ should be approximately unity. High fluorophore labelling density decreases this contrast, due to bridge artefacts.

The fluorophore density in the raw image data is estimated by the FCS number density metric, which is presented in Section 3.4. For all three of the distinct regions of the simulated crossed line object, the objective measures of whether the reconstructed images are free from bridge artefacts are correlated with a low value of the FCS number density metric.

\subsubsection{The effect of bridge artefacts in 3D-localization}

When two fluorophores generate an overlapping image on a camera, the signal may be misinterpreted not only as one coming from a single fluorophore in the wrong place, but also, the overlapping image will appear to be a 
single PSF that is elongated in the direction connecting the two real fluorophores. This is an important problem that can affect some of the 3-dimensional localization methods, such as the astigmatic method in which the ellipticity of the observed PSF is used to infer the z-position of the fluorophore. The elongation of the overlapping PSFs may lead to a noteworthy artefact in images of (say) actin filaments or straight fibrils, in which all the overlapping PSFs on the specimen will tend to be elongated in the same direction, along the length of the filament. This can cause an artefact in the reconstructed images, in which filaments oriented in one lateral direction can appear to be located on one side of the focal plane (say, above the focal plane), and perpendicular filaments may wrongly be interpreted as lying on the other side (i.e. below the focal plane), even if in reality they rest at identical z-positions.

\subsection{Metrics of image quality in localization-based super-resolution microscopy}

\subsubsection{Metrics of precision, as a test for blurred super-resolution images}

In general the performance of a localization microscope can be assessed in terms of its localization accuracy. Quantitatively, this is the difference between the inferred positions of fluorophores and their true positions. The list of inferred fluorophore positions may contain spurious additional entries associated with bridge artefacts or noise; or it may be missing some entries; or in the simplest case the inferred positions may simply have some finite error. Positional error can be decomposed into factors which are static or stochastic. Static factors lead to identical errors if a fluorophore in the same place is repeatedly measured. Such factors include optical system biases such as chromatic offset in two color imaging, and tend to produce a super-resolution image which is displaced or a distorted representation of the specimen. Stochastic factors produce random errors in the estimated fluorophore position: these lead to blurring of the reconstructed image, and therefore they fundamentally limit localization microscope resolution, although random positional errors are not the only limit on resolution. There are two fundamental sources of random localization errors, which are (a) the stochastic nature of photon detection, and (b) the finite signal to noise achieved on the camera. There are two widely used formulas for estimating the random variance of fluorophore localization due to these factors, by Thompson(Eq. 1) and Mortensen(Eq. 2).[29, 31]

$$
\begin{gathered}
\left\langle\left(\Delta x^{2}\right)\right\rangle_{T}=\frac{\sigma^{2}+a^{2} / 12}{N}+\frac{8 \pi \sigma^{4} b^{2}}{a^{2} N^{2}} \\
\left\langle\left(\Delta x^{2}\right)\right\rangle_{M}=\frac{\sigma^{2}+a^{2} / 12}{N}\left(\frac{16}{9}+\frac{8 \pi\left(\sigma^{2}+a^{2} / 12\right)^{2} b^{2}}{N a^{2}}\right),
\end{gathered}
$$

where $N$ is the number off collected photons, $b$ is the photon background of the image in the units of photons/pixel/frame, $\sigma$ is the standard deviation of the Gaussian shaped PSF spot, $a$ is the pixel size in the sample space. These can easily be evaluated from raw image data. Ober [30] also analysed the dependence of the localization precision as function of pixel size and relative position of the molecule in the raw image pixel. DeSantis developed a similar empirical formulation for estimating the variance of the sigma parameter estimation based on the same assumptions made by Thompson [51]. 


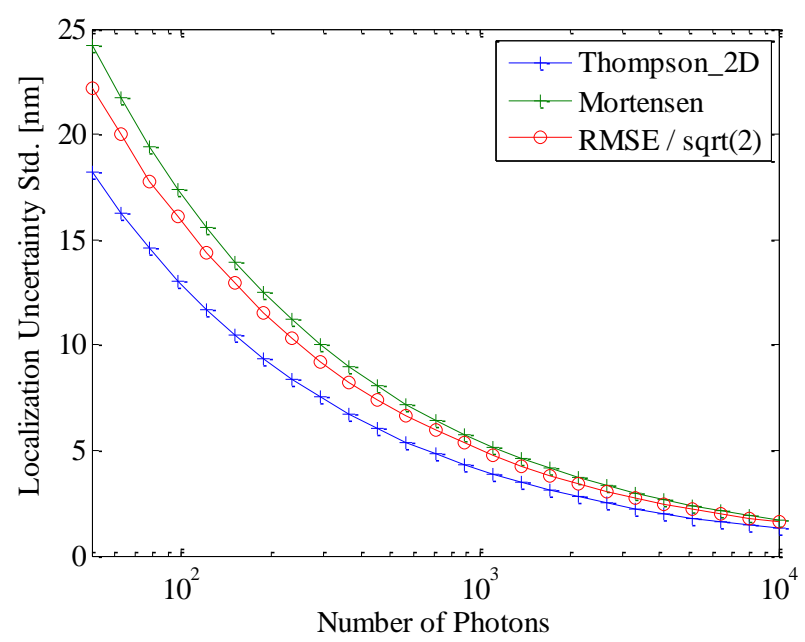

Figure 12. Localization uncertainty estimated from raw image data by the Thompson and Mortensen formulas are broadly consistent with the empirically measured spread of localised positions determined by simple PSF

fitting algorithms for static single molecule samples, provided complex problems such as non-uniform background noise are not significant.

Fig. 12 compares the standard deviations predicted by these two formulas with the root mean square error (RMSE) of a sparse Gaussian fitting algorithm (rainSTORM localization software) obtained by repeated simulations of an individual static molecule generated by testSTORM with identical parameters as in Section 3.3. The formulas provide good estimates of stochastic localization error, although neither these equations nor simple simulations can capture complex sources of error such as non-uniform or time-varying fluorescent background signals. Nonetheless, they are important metrics of super-resolution image quality, because they are directly related to the resolution of reconstructed image. In the idealised case that resolution is only limited by localisation precision (and not by a limited number of fluorescent labels, or label size, specimen movement, inhomogenous background, or other factors), it can be shown that the resolution of the localisation microscope is two times the mean localisation precision given by one of these formulas [27]. Features in a reconstructed image that are much larger than this limit may therefore be interpreted as distinct objects, and a small value for the mean precision estimate for raw image data is a desirable metric.

\subsubsection{A metric of fluorophore density, as a test for Bridge artefacts}

The quality of a localization microscope image depends on the photoswitching fluorescent molecule images being spatially sparse enough for accurate analysis. Analysing excessively dense fluorophore images can generate the bridge artefacts and edge artefacts discussed in 3.3, which are spurious structures that do not exist on the specimens. In order to be sure that super-resolution images are robust against such artefacts, it is important to have a metric of fluorophore density in the raw data. Fortunately, localization microscope measurements can be analysed to estimate the density of active fluorophores, using a technique based on fluorescence correlation spectroscopy (FCS) [52] which is outlined in Fig. 13. 

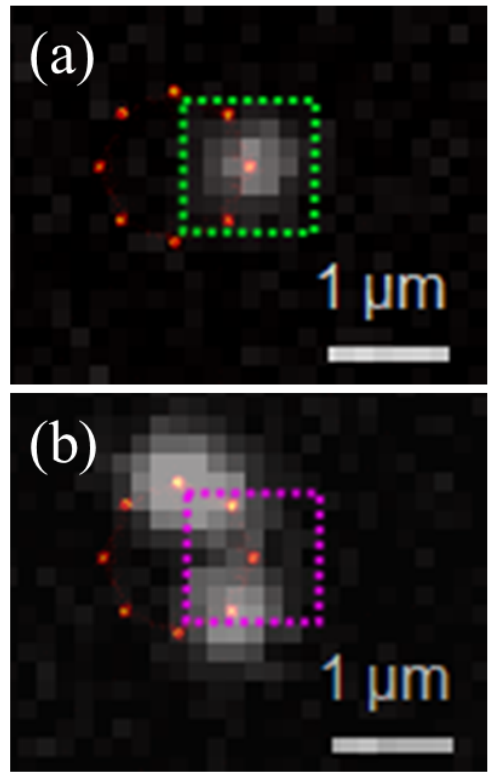
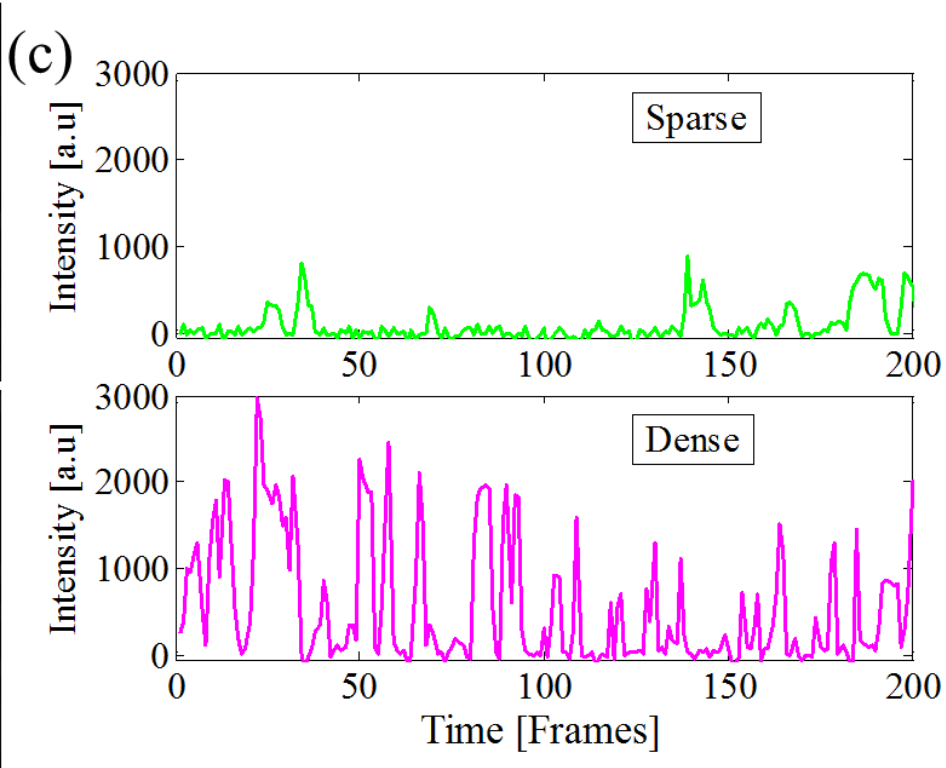

Identify areas containing fluorophores

Find number density for each fitting area from fluorescence correlation

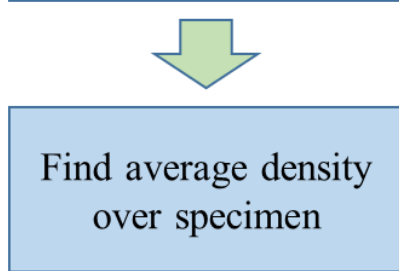

Figure 13. A number density metric for localisation microscopy can be evaluated by building on fluorescence correlation spectroscopy (FCS). A single frame of camera data is shown in grey for specimens producing (a) sparse, and (b) partially overlapping photoswitching images, and the correctly super-resolved structure of the specimens are shown overlaid in red. For each region containing significant amounts of localisations, a box is defined which encompasses the area of raw image data that is used for fitting the fluorophore positions (7x 7 pixels in this case). The total fluorescence intensity in these boxed regions is plotted throughout the duration of the image stack (c). The relative mean square fluctuation of total intensity is inversely proportional to the average number of fluorophores in the fitting region, and this can be identified for different regions of the specimen or averaged as necessary.

In fluorescence correlation spectroscopy, the relative mean square fluctuation of the fluorescence signal reaching a point-detector, $i$, is inversely proportional to the average number of fluorophores in the observation volume, $N$, (Eq. 3).

$$
\frac{\operatorname{Var}(i)}{<i>^{2}}=1 / N
$$

Where $\langle i>$ is the mean fluorescence signal and $\operatorname{Var}(i)$ is its variance, and $i$ can be measured as a discrete, timeresolved fluorescence intensity. This result was established in confocal fluorescence correlation spectroscopy, typically for the purpose of estimating the absolute number density of fluorophores dissolved in a fluid and undergoing diffusion or flow through the measurement volume. It is generally true for measurements of fluorescent solutions with randomly-distributed fluorophores in which the number of fluorophores being detected is Poisson-distributed. In localization microscopy, it is commonly assumed that the random kinetics of photoswitching fluorescent molecules leads to Poisson-distributed numbers of active fluorophores. It is therefore possible to estimate $N$ in the raw image data using Eq.3, even though the changes in the fluorescence signal are caused by blinking and not by movement. Thus the variation of the fluorescent signal can be used to estimate the number of molecules in a given imaged area. This is the principle of how to use FCS analysis to estimate the density of fluorophores in localization microscopy.

Typically, sparse localisation microscopy algorithms attempt to infer fluorophore positions by fitting to image data within a region of the camera (e.g. a 7x7 pixel square centred on a local maxima). A metric of fluorophore density needs to estimate the average number density of fluorophores in regions of this size. Intuitively, to avoid overlapping images leading to bridge artefacts, this number should be significantly less than 1 . In fact, based on the occurrence of bridge artefacts in Figures 8 and 9, it can be seen that bridge artefacts consistently 
become significant with sparse localisation algorithms when the value of this metric exceeds about 0.2. This critical value recurs for simulations of various specimen geometries, and hence the FCS number density is a valuable metric for assuring the quality of localisation microscopy images of unknown specimens.

\subsubsection{Resolution limit by the dye blinking.}

The imaging performance of the localization microscope strongly depends on the sparseness of the image data. There are two major categories of localization algorithms: single molecule fitting and multiple molecule fitting methods [53]. The former are simpler [54], and are based on the strict prerequisite fluorophore images do not overlap spatially in the raw image data. This means they can be segmented and analysed individually. The multiple molecule fitting algorithms, such as DAOSTORM [55], can handle denser fluorophore images up to a higher cutoff density that can be determined using simulated data. In the second case (fitting to overlapped molecules), the algorithm tries to fit the candidate area of a frame with more than one PSF spots. This approach increases the recall rate of the localization algorithm, but the localization precision for one molecule decreases since the overlapping PSF spots are appearing as an additional noise source for each other from the PSF spot fitting point of view. Ideally, therefore, the sparseness of the raw image data has to be controlled to avoid the overlapping PSF spots. Wolter et. al. analysed the performance of single molecule fitting algorithms by means of assessing the recall rate as a function spot density on a single frame. They found that the number of detected spots have a maximum around 0.7 spots per frame per $\mu \mathrm{m}^{2}$. [56]

The dye parameters have a strong influence on the probability of PSF overlapping. The blinking of the dyes used in dSTORM imaging has the following main parameters: on- and off-state lifetime, number of detected photons and duty cycle. Duty cycle (DC) is the ratio of the on-state lifetime and the sum of on- and off-state lifetimes.

The maximum number of molecules placed in a PSF area could be estimated in the following way to keep satisfied the condition of low probability of PSF overlapping. If the molecules in the PSF area are sequentially turned on for exactly a duration of the expected value of the on-state lifetime, then the maximum number of molecules which do not overlap is equal to the reciprocal of the duty cycle. The size of smallest resolvable object can be determined by inserting the PSF size and maximum number of molecules in the PSF area into the Nyquist sampling theorem. Therefore the duty cycle also set a limit to the size of the smallest object which could be resolved at given blinking parameters. This size strongly depends on the object geometry, the labelling homogeneity and the labelling density (dye molecules per antibody). The minimum resolvable object size could be estimated in case of homogeneously labelled line like sample types as the double of the ratio of the PSF width and the maximum number of molecules per PSF, which can be written as:

$$
d_{\min , 1 D} \approx 2 \cdot \frac{P S F_{w}}{N_{\max }}=2 \cdot P S F_{w} \cdot D C
$$

The definition of the PSF width is a somewhat subjective task, it might depend on the used localization algorithm. In 2D the minimum size depends on the square root of the area of the PSF, which could be written as:

$$
d_{\min , 2 D} \approx 2 \cdot \sqrt{\frac{P S F_{A}}{N_{\max }}}=2 \cdot \sqrt{P S F_{A} \cdot D C}
$$

Therefore, the blinking parameters of the dye also define a resolution limit for the super-resolved image if the non-overlapping condition is satisfied. Thus, there are two limiting factors for single molecule localization algorithms: the localization precision for one blinking event mainly limited by the collected number of photons and the minimum resolvable object size determined by the PSF size and the duty cycle of the dye. Linde et. al. analysed the effect of duty cycle on the image quality by means of simulation. They results also show that increasing the duty cycle leads to artefacts. [57] 
To further improve image quality the type of limitation has to be determined. If the duty cycle of dye is low enough then the stochastic nature of localization is the main limiting factor. In this case the variance of localization has to be decreased. The number of collected photons is determined mainly by the fluorescent dye. Thus in this case the dye shall be replaced. On the other hand when the limiting factor is the duty cycle of the dye, then more optimization opportunities are available. First, increasing the intensity of the excitation laser the on-state lifetime of the dye could be decreased while maintaining the number of collected photons. The limit is the maximum laser power output of the system and the photo-damage of the sample. If one of them is reached, then the composition of the buffer solution can be altered to change the life time of the on-off states.

\section{Conclusions}

Localization-based super-resolution microscopy methods can improve the spatial resolution due to the precise localization of individual fluorescent molecules. From the hardware point of view such systems are modified traditional microscopic systems originally optimized for diffraction limited imaging. Therefore, minor effects typically neglected in traditional microscopy start to play important roles, and the implementation of the final high resolution images is sometimes challenging. In this chapter imaging artefacts were categorized by their origin: errors introduced by the optical components, the sample or by the algorithms. It was shown how monochromatic and chromatic aberrations, in focus and defocused arrangements, on-axis and off-axis position of the molecules affected the final image quality. We then presented methods for calculating metrics of superresolution image quality. The open-source testSTORM software can be used to simulate arrays of superresolution image data based on common specimen geometries. Simulation arrays are useful for optimising experimental parameters provided a metric of image quality is available for ranking the results. We showed that objective quality metrics can easily be designed for classifying the super-resolution images obtained from simulated data when the underlying specimen is known, and that these objective metrics are consistent with subjective visual impression of whether the super-resolution image is of high quality and free from artefacts. We then described two useful image quality metrics which can be used for blind-assessment of super-resolution image quality when nothing is known about the specimen. The mean localisation precision is based on the Thompson or Mortensen equations, and this metric is known to be related to the random blurring of superresolved localisation microscopy images, and it is one factor that directly limits localisation microscope resolution (although not the only factor). This metric is valuable for identifying whether limited photoncollection leads to poor precision and loss of resolution. The FCS number-density metric of active fluorophore density can readily be evaluated from raw image data, and provides a valuable indication of whether overlapping fluorophore images may lead to bridge artefacts in the super-resolved images. This second metric is useful for testing whether high-density fluorophore images may lead to unreliable image reconstructions. In combination, these quality metrics and the knowledge of the optical system limitations are very helpful for assuring the robust and precise interpretation of super-resolved localisation microscopy images. It is worth noting here, that image quality metrics typically optimistically overestimate the practical, experimentally available resolution limit, due to system and sample imperfections. In case of highly structured, densely labelled and thick samples, estimation of spatial resolution based on theoretical considerations is difficult.

\section{Acknowledgement}

The research was partially funded by the Hungarian Brain Research Program (KTIA_13_NAP-A-I/14), the Marie Curie Integration Grant (PCIG13-GA-2013-618273), the EPSRC and the János Bolyai Research Scholarship of the Hungarian Academy of Sciences. We acknowledge Lambda Research Corporation for providing the OSLO optical system design software for the simulations. 


\section{References}

[1] B.R. Masters, History of the Optical Microscope in Cell Biology and Medicine, Encyclopedia of Life Sciences. (2001).

[2] R. Heintzmann, G. Ficz, Breaking the resolution limit in light microscopy, Briefings in Functional Genomics and Proteomics. 5 (2006) 289-301.

[3] M. Born, E. Wolf, Principles of optics, Cambridge University Press, 2002

[4] S.W. Hell, Far-Field Optical Nanoscopy, Science. 316 (2007) 1153-1158.

[5] S.W. Hell, J. Wichmann, Breaking the diffraction resolution limit by stimulated emission: stimulatedemission-depletion fluorescence microscopy, Optics Letters. 19 (1994) 780.

[6] T.A. Klar, S.W. Hell, Subdiffraction resolution in far-field fluorescence microscopy, Optics Letters. 24 (1999) 954.

[7] M. G. Gustafsson, Surpassing the lateral resolution limit by a factor of two using structured illumination microscopy, J Microsc198 (Pt 2) (2000) 82-87.

[8] M.G.L.Gustafsson, Nonlinear structured-illumination microscopy: Wide-field fluorescence imaging with theoretically unlimited resolution, Proceedings of the National Academy of Sciences. 102 (2005) 13081-13086.

[9] E. Betzig, G.H. Patterson, R. Sougrat, O.W. Lindwasser, S. Olenych, J.S. Bonifacino, et al.,Imaging Intracellular Fluorescent Proteins at Nanometer Resolution, Science. 313 (2006) 1642-1645.

[10] S.T. Hess, T.P.K. Girirajan, M.D. Mason, Ultra-High Resolution Imaging by Fluorescence Photoactivation Localization Microscopy, Biophysical Journal. 91 (2006) 4258-4272.

[11] M.J. Rust, M. Bates, X. Zhuang, Sub-diffraction-limit imaging by stochastic optical reconstruction microscopy (STORM), Nature Methods. 3 (2006) 793-796.

[12] B. Huang, H. Babcock, X. Zhuang, Breaking the Diffraction Barrier: Super-Resolution Imaging of Cells, Cell. 143 (2010) 1047-1058.

[13] S. van de Linde, A. Löschberger, T. Klein, M. Heidbreder, S. Wolter, M. Heilemann, et al., Direct stochastic optical reconstruction microscopy with standard fluorescent probes, Nat Protoc. 6 (2011) 991-1009.

[14] L. Novotny, B. Hecht, Principles of Nano-Optics, Second Edition, Cambridge University Press, 2012

[15] S. Stallinga, B. Rieger, Accuracy of the Gaussian Point Spread Function model in 2D localization microscopy, Optics Express. 18 (2010) 24461.

[16] M.D. Lew, M.P. Backlund, W.E. Moerner, Rotational Mobility of Single Molecules Affects Localization Accuracy in Super-Resolution Fluorescence Microscopy, Nano Lett. 13 (2013) 3967-3972.

[17] G. T. Dempsey, W. Wang, X. Zhuang, Fluorescence imaging at sub-diffraction-limit resolution with stochastic optical reconstruction microscopy, Handbook of single-molecule biophysics, Springer Science+Business Media, 2009 
[18] P. Annibale, M. Scarselli, M. Greco, A. Radenovic, Identification of the factors affecting co-localization precision for quantitative multicolor localization microscopy, Optical Nanoscopy. 1 (2012) 9.

[19] Lambda Research Corp., OSLO optics software, optics reference ver. 6.1. 2001.

[20] M. Mandai and K. Yamaguchi, "Immersion microscope objective lens", US patent US 7,046,451 B2 (2006).

[21] H.H. Hopkins, M.J. Yzuel, The Computation of Diffraction Patterns in the Presence of Aberrations, Optica Acta: International Journal of Optics. 17 (1970) 157-182.

[22] M. Erdelyi, E. Rees, D. Metcalf, G.S.K. Schierle, L. Dudas, J. Sinko, et al., Correcting chromatic offset in multicolor super-resolution localization microscopy, Optics Express. 21 (2013) 10978.

[23] J. Sinkó, R. Kákonyi, E. Rees, D. Metcalf, A.E. Knight, C.F. Kaminski, et al., TestSTORM: Simulator for optimizing sample labeling and image acquisition in localization based super-resolution microscopy, Biomedical Optics Express. 5 (2014) 778.

[24] http://titan.physx.u-szeged.hu/ adoptim/

[25] R. Henriques, M. Lelek, E.F. Fornasiero, F. Valtorta, C. Zimmer, M.M. Mhlanga, QuickPALM: 3D realtime photoactivation nanoscopy image processing in ImageJ, Nature Methods. 7 (2010) 339-340.

[26] S. Wolter, M. Schüttpelz, M. Tscherepanow, S. Van De Linde, M. Heilemann, M. Sauer, Real-time computation of subdiffraction-resolution fluorescence images, Journal of Microscopy. 237 (2010) 12-22.

[27] E.J. Rees, M. Erdelyi, D. Pinotsi, A. Knight, D. Metcalf, C.F. Kaminski, Blind assessment of localisation microscope image resolution, Optical Nanoscopy. 1 (2012) 12.

\section{[28] http://laser.cheng.cam.ac.uk/wiki/index.php/Resources}

[29] K.I. Mortensen, L.S. Churchman, J.A. Spudich, H. Flyvbjerg, Optimized localization analysis for singlemolecule tracking and super-resolution microscopy, Nature Methods. 7 (2010) 377-381.

[30] R.J. Ober, S. Ram, E.S. Ward, Localization Accuracy in Single-Molecule Microscopy, Biophysical Journal. 86 (2004) 1185-1200.

[31] R.E. Thompson, D.R. Larson, W.W. Webb, Precise Nanometer Localization Analysis for Individual Fluorescent Probes, Biophysical Journal. 82 (2002) 2775-2783.

[32] C. Steinhauer, R. Jungmann, T. Sobey, F. Simmel, P. Tinnefeld, DNA Origami as a Nanoscopic Ruler for Super-Resolution Microscopy, Angewandte Chemie International Edition. 48 (2009) 8870-8873.

[33] R. Jungmann, C. Steinhauer, M. Scheible, A. Kuzyk, P. Tinnefeld, F.C. Simmel, Single-Molecule Kinetics and Super-Resolution Microscopy by Fluorescence Imaging of Transient Binding on DNA Origami, Nano Lett. 10 (2010) 4756-4761. 
[34] S. van de Linde, U. Endesfelder, A. Mukherjee, M. Schüttpelz, G. Wiebusch, S. Wolter, et al., Multicolor photoswitching microscopy for subdiffraction-resolution fluorescence imaging, Photochemical \& Photobiological Sciences. 8 (2009) 465.

[35] I. Testa, C.A. Wurm, R. Medda, E. Rothermel, C. von Middendorf, J. Fölling, et al., Multicolor Fluorescence Nanoscopy in Fixed and Living Cells by Exciting Conventional Fluorophores with a Single Wavelength, Biophysical Journal. 99 (2010) 2686-2694.

[36] D. Baddeley, D. Crossman, S. Rossberger, J.E. Cheyne, J.M. Montgomery, I.D. Jayasinghe, et al., 4D Super-Resolution Microscopy with Conventional Fluorophores and Single Wavelength Excitation in Optically Thick Cells and Tissues, PLoS ONE. 6 (2011) e20645.

[37] L.S. Churchman, J. A. Spudich, Colocalization of fluorescent probes: accurate and precise registration with nanometer resolution, Single-molecule techniques: a Laboratory Manual, PR Selvin and TJ Ha, eds., 73-84., 2007.

[38] R. McGorty, J. Schnitzbauer, W. Zhang, B. Huang, Correction of depth-dependent aberrations in 3D single-molecule localization and super-resolution microscopy, Optics Letters. 39 (2014) 275.

[39] M. Erdelyi, E. Rees, D. Metcalf, G.S.K. Schierle, L. Dudas, J. Sinko, et al., Correcting chromatic offset in multicolor super-resolution localization microscopy, Optics Express. 21 (2013) 10978.

[40] A. Pertsinidis, Y. Zhang, S. Chu, Subnanometre single-molecule localization, registration and distance measurements, Nature. 466 (2010) 647-651.

[41] J. Pawley, Handbook of Biological Confocal Microscopy, 3rd ed., Springer, New York, 2006, Chapter 8, 11.

[42] S. Proppert, S. Wolter, T. Holm, T. Klein, S. van de Linde, M. Sauer, Cubic B-spline calibration for 3D super-resolution measurements using astigmatic imaging, Optics Express. 22 (2014) 10304.

[43] I. Izeddin, M. El Beheiry, J. Andilla, D. Ciepielewski, X. Darzacq, M. Dahan, PSF shaping using adaptive optics for three-dimensional single-molecule super-resolution imaging and tracking, Optics Express. 20 (2012) 4957.

[44] D. Axelrod, Total internal reflection fluorescence microscopy, in: P. Torok, F. J. Kao (Eds.), Optical Imaging and Microscopy, Vol. 87 of Springer Series in Optical Sciences, Springer Verlag, New York, 2007, Chapter 8.

[45] C.A. Konopka, S.Y. Bednarek, Variable-angle epifluorescence microscopy: a new way to look at protein dynamics in the plant cell cortex, The Plant Journal. 53 (2008) 186-196.

[46] M. Tokunaga, N. Imamoto, K. Sakata-Sogawa, Highly inclined thin illumination enables clear singlemolecule imaging in cells, Nature Methods. 5 (2008) 159-161.

[47] J. Sinkó, G. Szabó, M. Erdélyi, Ray tracing analysis of inclined illumination techniques, Optics Express. 22 (2014) 18940.

[48] S. Jakobs, C.A. Wurm, Super-resolution microscopy of mitochondria, Current Opinion in Chemical Biology. 20 (2014) 9-15. 
[49] L A. Loschberger, S. van de Linde, M.-C. Dabauvalle, B. Rieger, M. Heilemann, G. Krohne, et al., Superresolution imaging visualizes the eightfold symmetry of gp210 proteins around the nuclear pore complex and resolves the central channel with nanometer resolution, Journal of Cell Science. 125 (2012) 570-575.

[50] G.T. Dempsey, J.C. Vaughan, K.H. Chen, M. Bates, X. Zhuang, Evaluation of fluorophores for optimal performance in localization-based super-resolution imaging, Nature Methods. 8 (2011) 1027-1036.

[51] M.C. DeSantis, S.H. DeCenzo, J.-L. Li, Y.M. Wang, Precision analysis for standard deviation measurements of immobile single fluorescent molecule images, Optics Express. 18 (2010) 6563.

[52] D. Magde, W.W. Webb, E.L. Elson, Fluorescence correlation spectroscopy. III. Uniform translation and laminar flow, Biopolymers. 17 (1978) 361-376.

[53] A. Small, S. Stahlheber, Fluorophore localization algorithms for super-resolution microscopy, Nature Methods. 11 (2014) 267-279.

[54] M.K. Cheezum, W.F. Walker, W.H. Guilford, Quantitative Comparison of Algorithms for Tracking Single Fluorescent Particles, Biophysical Journal. 81 (2001) 2378-2388.

[55] S.J. Holden, S. Uphoff, A.N. Kapanidis, DAOSTORM: an algorithm for high- density super-resolution microscopy, Nature Methods. 8 (2011) 279-280.

[56] S. Wolter, U. Endesfelder, S. van de Linde, M. Heilemann, M. Sauer, Measuring localization performance of super-resolution algorithms on very active samples, Optics Express. 19 (2011) 7020.

[57] S. van de Linde, S. Wolter, M. Heilemann, M. Sauer, The effect of photoswitching kinetics and labeling densities on super-resolution fluorescence imaging, Journal of Biotechnology. 149 (2010) 260-266. 
Figure 1
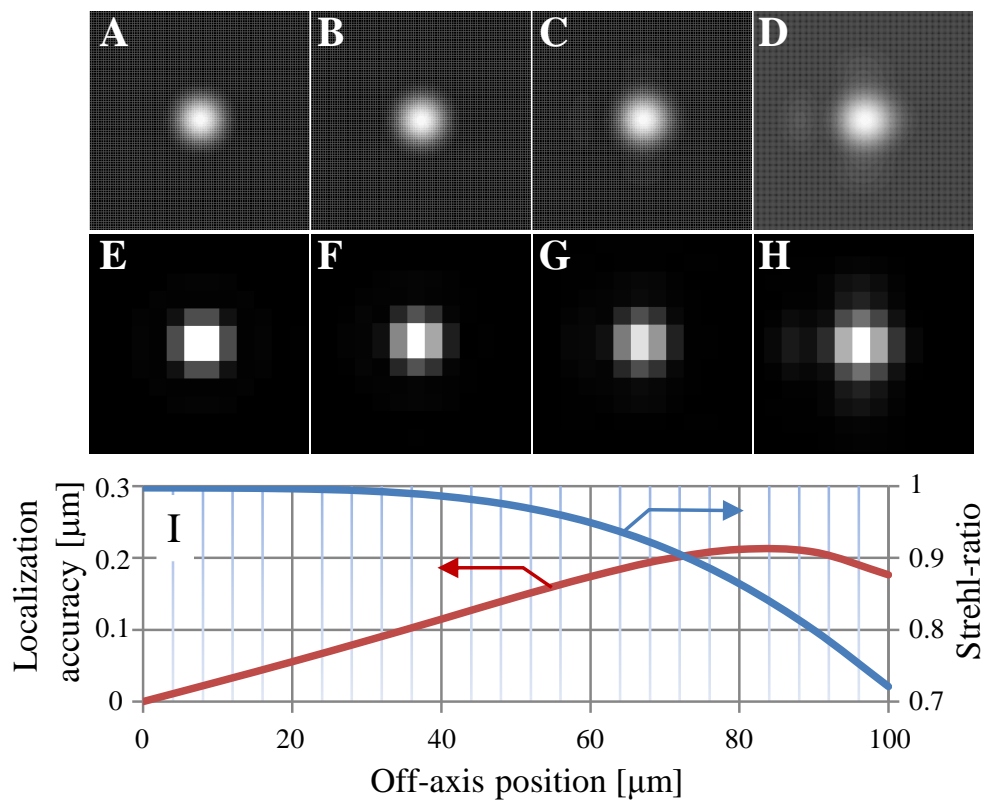

$$
\text { है }
$$

$8 \stackrel{\frac{2}{0}}{\frac{5}{2}}$ 

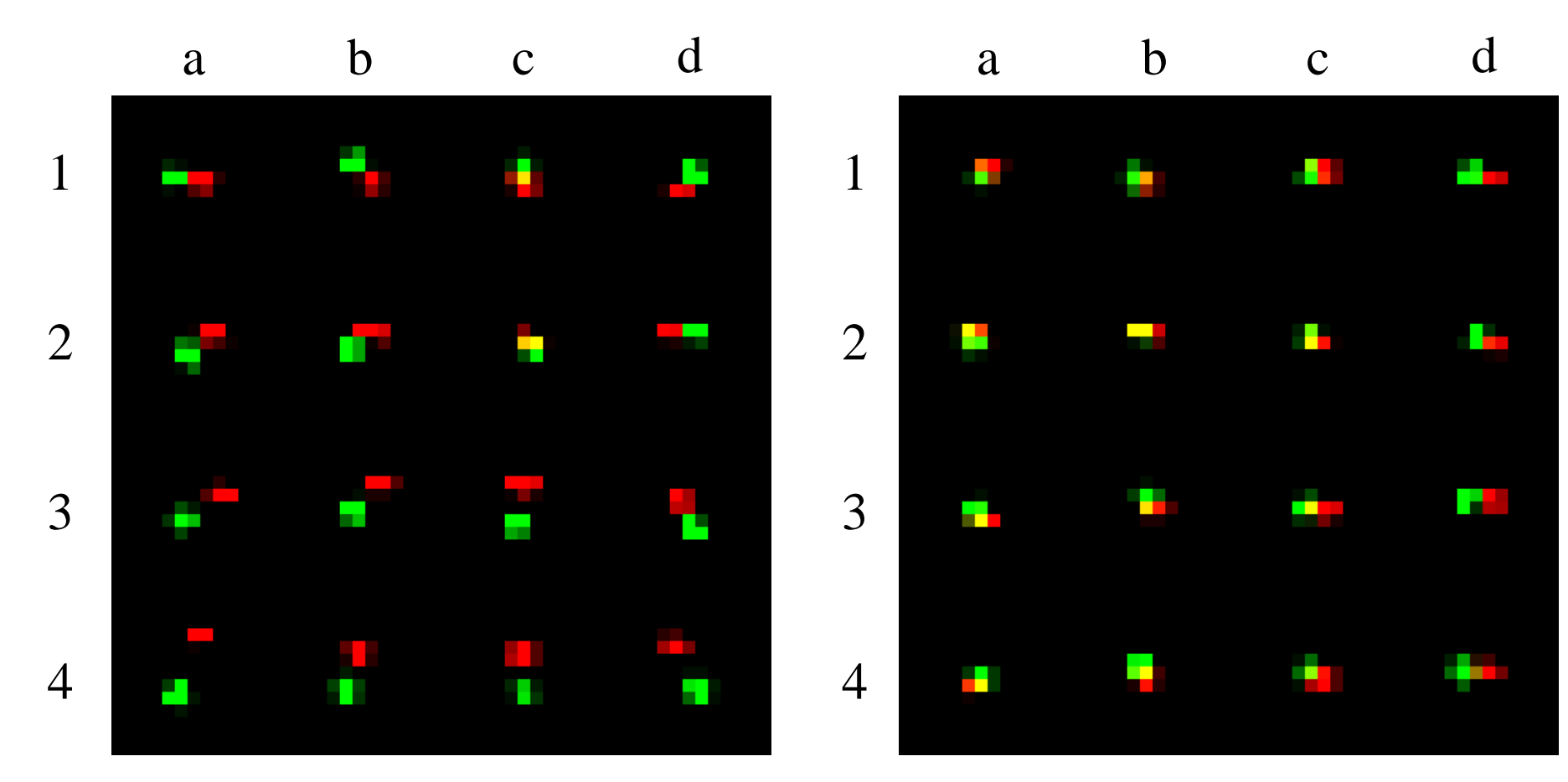

Figure 2 

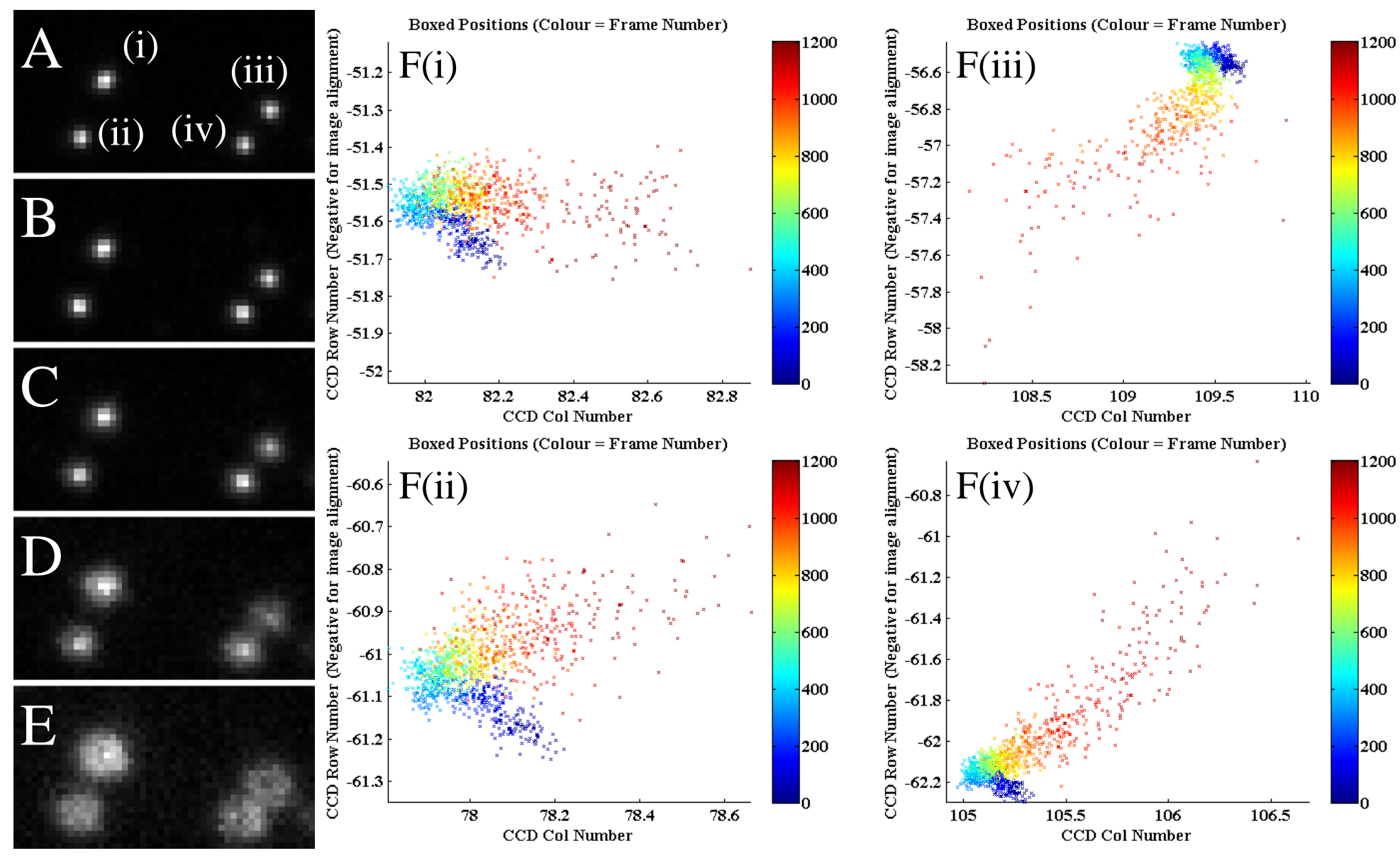


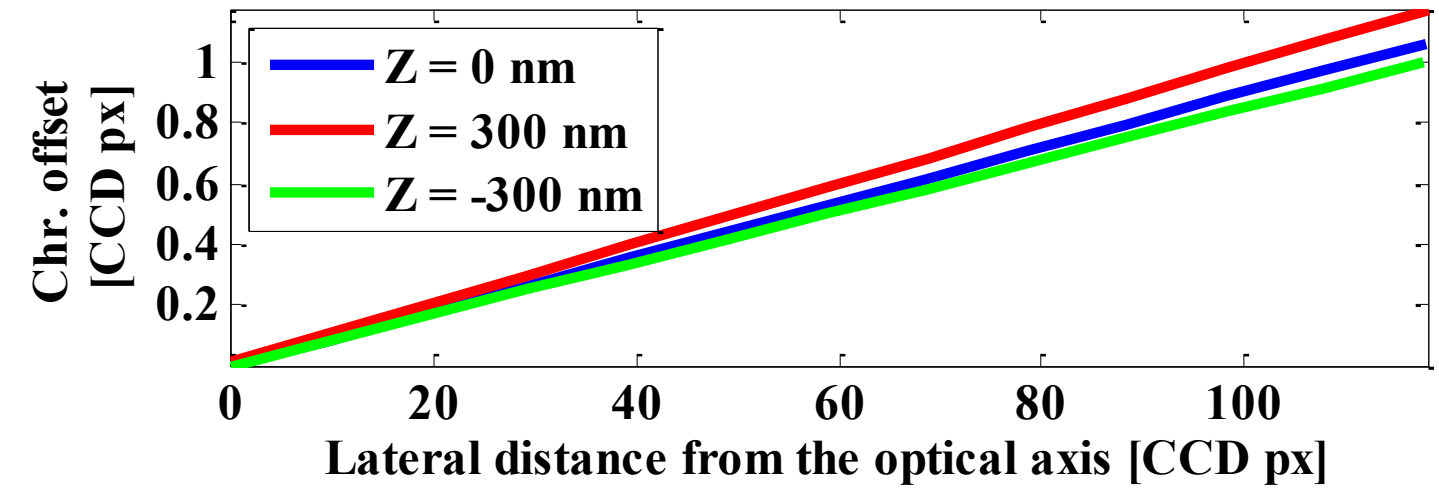




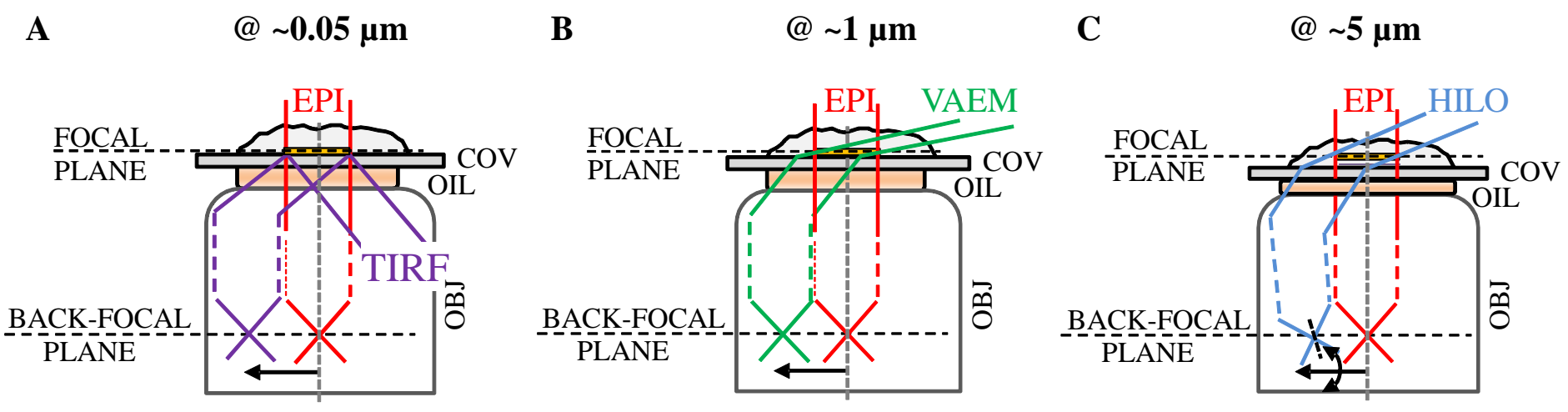


A

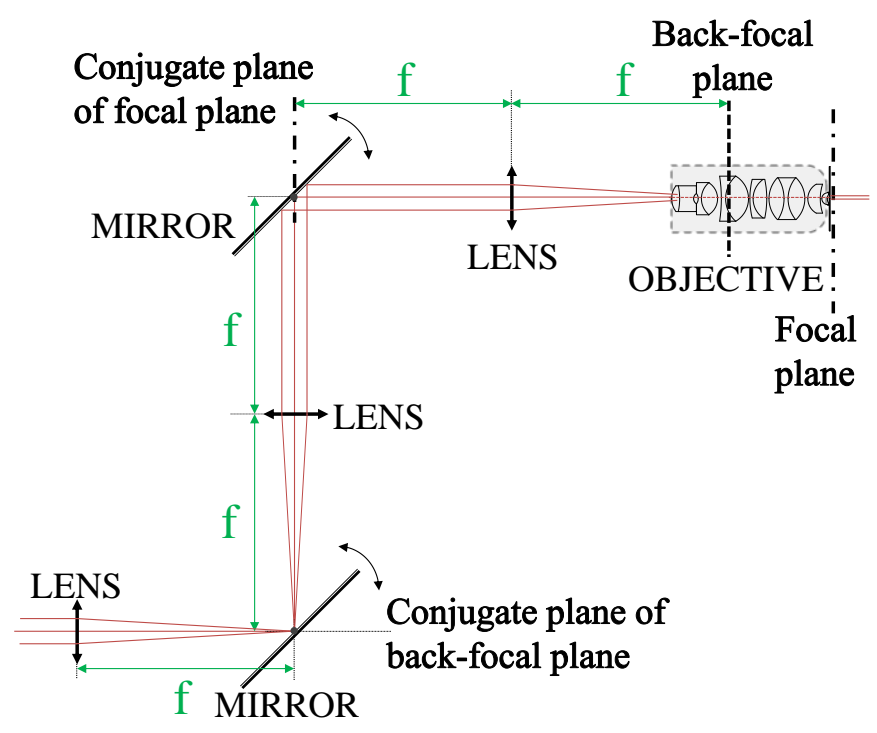

B

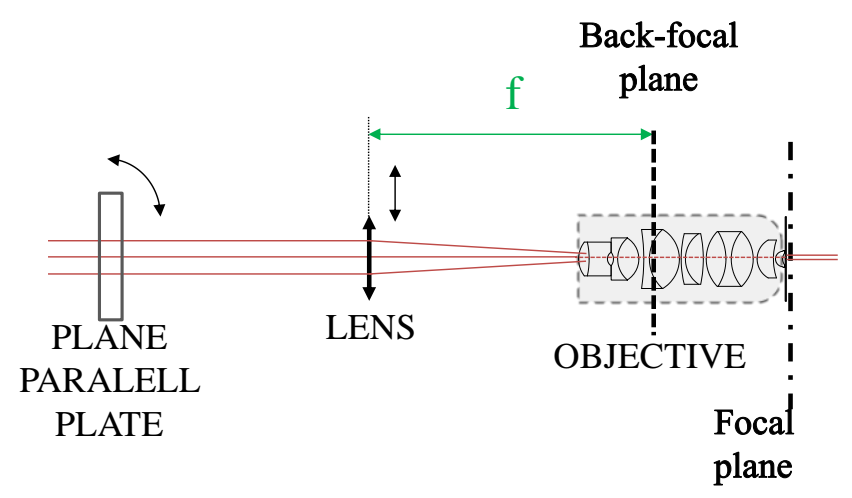




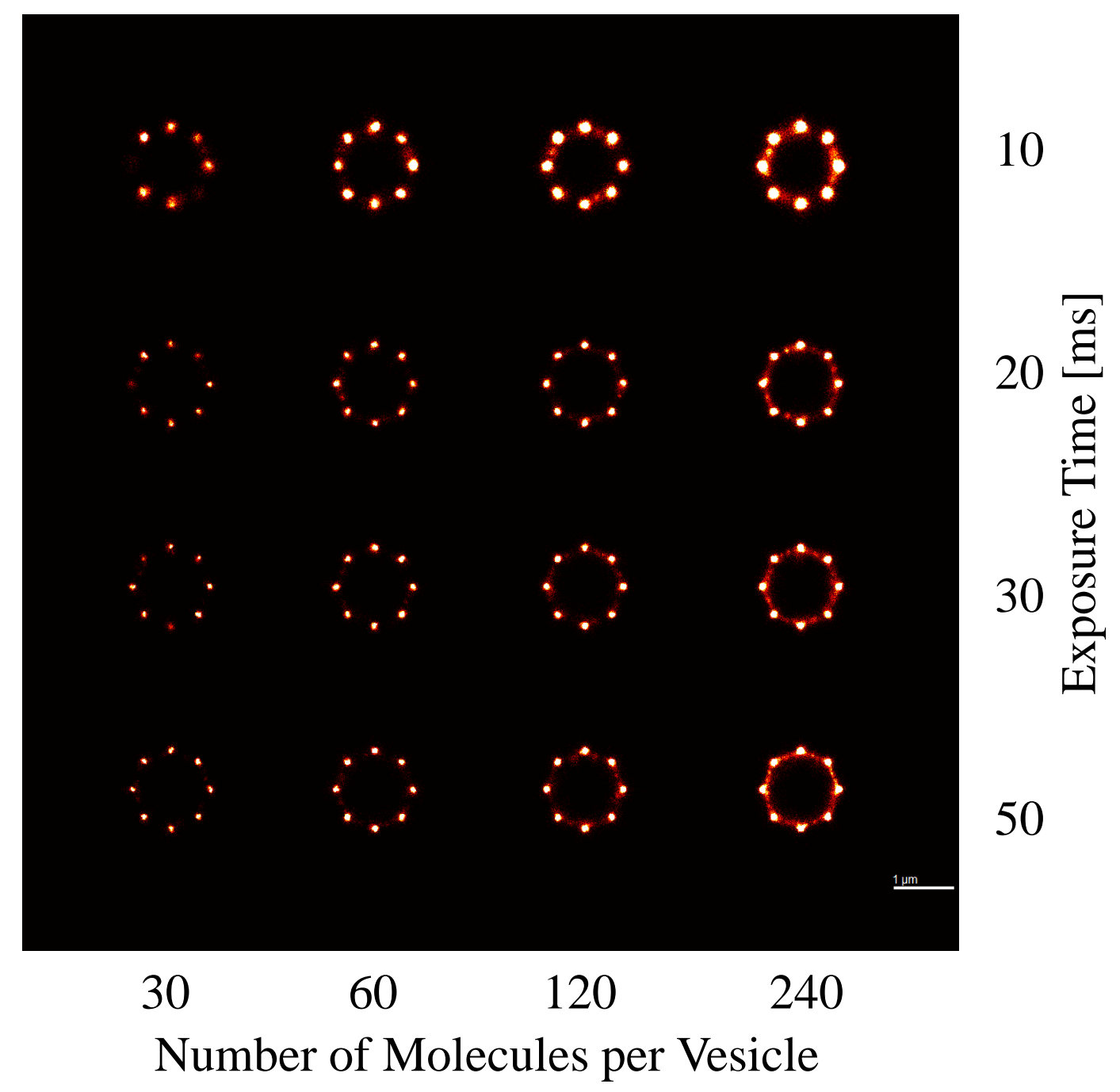




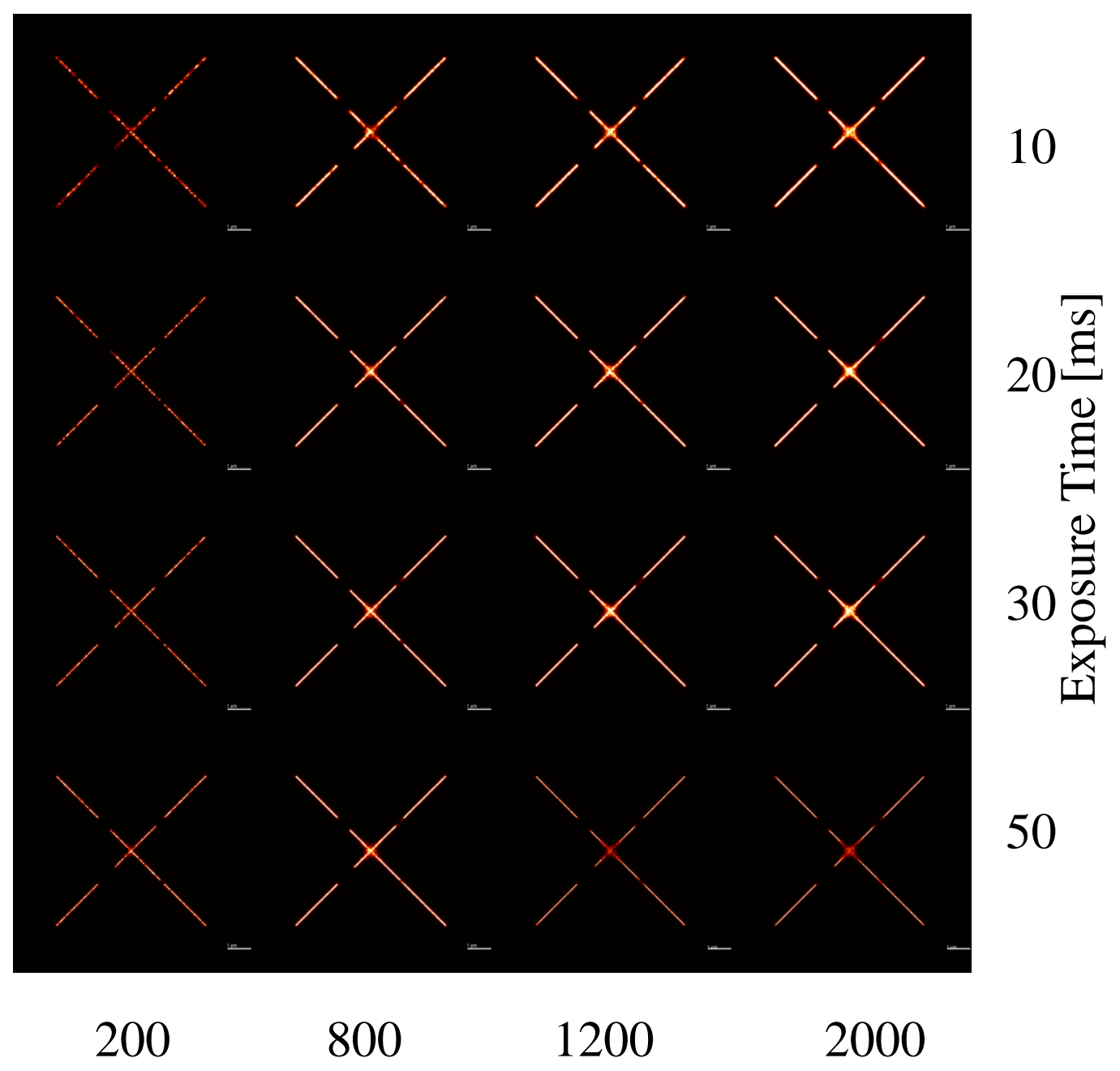

Labelling Density [Molecules / $\mu \mathrm{m}$ ] 


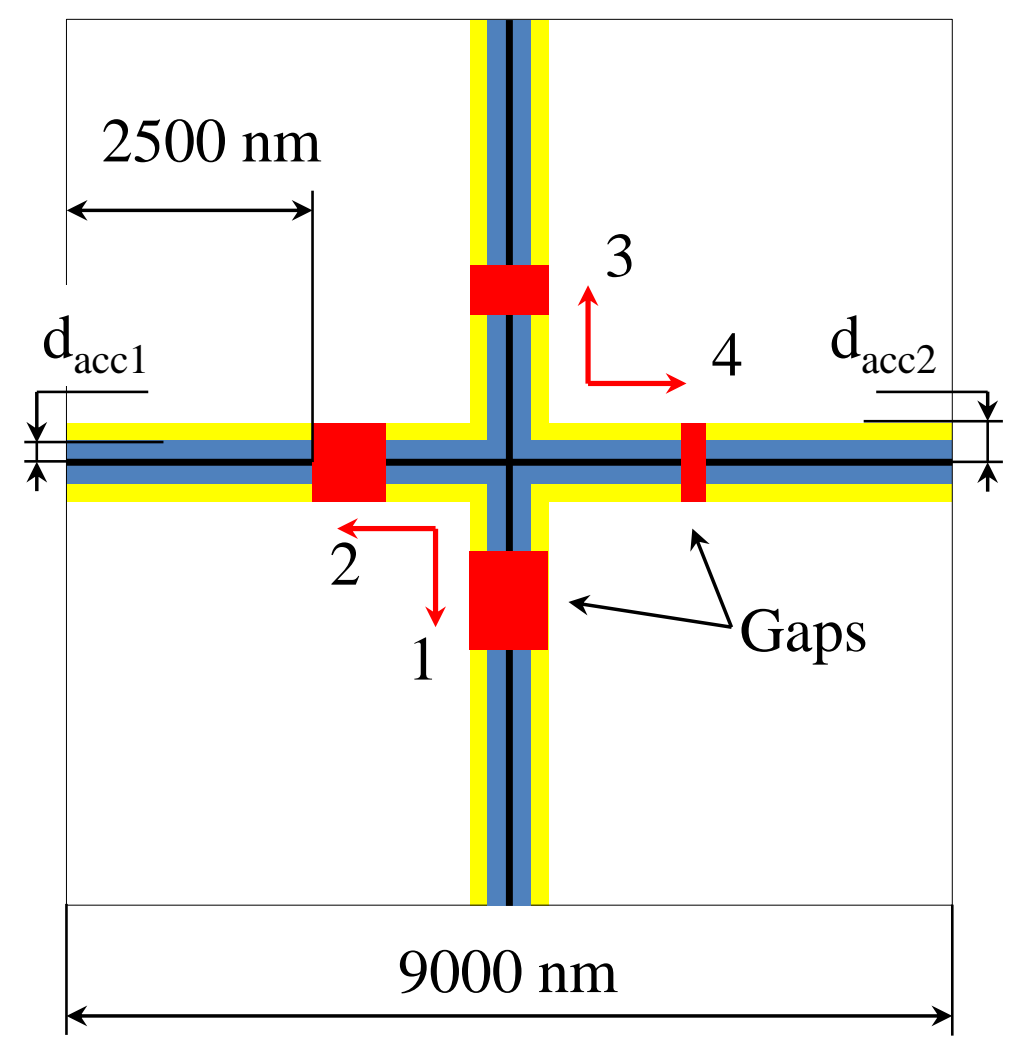




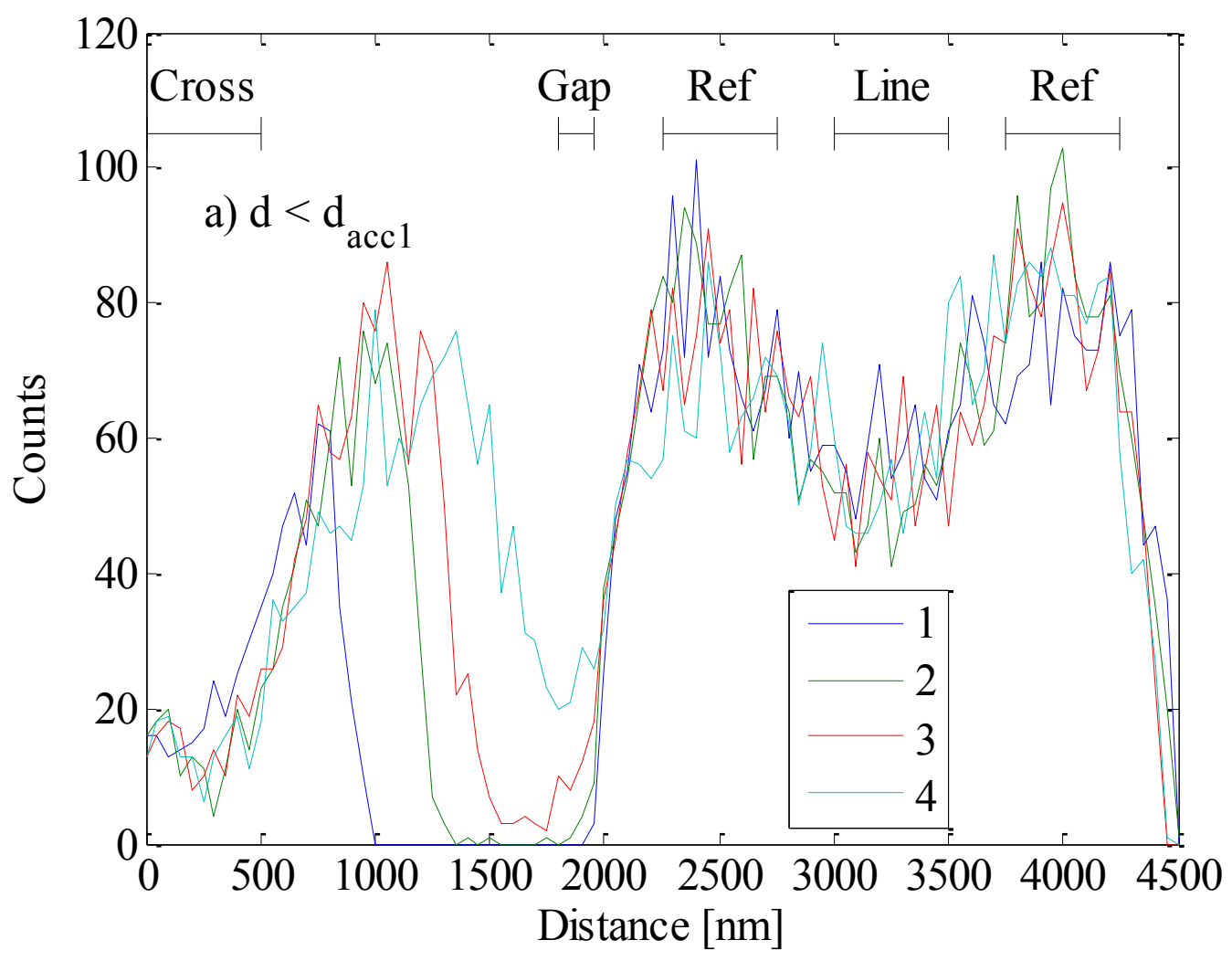




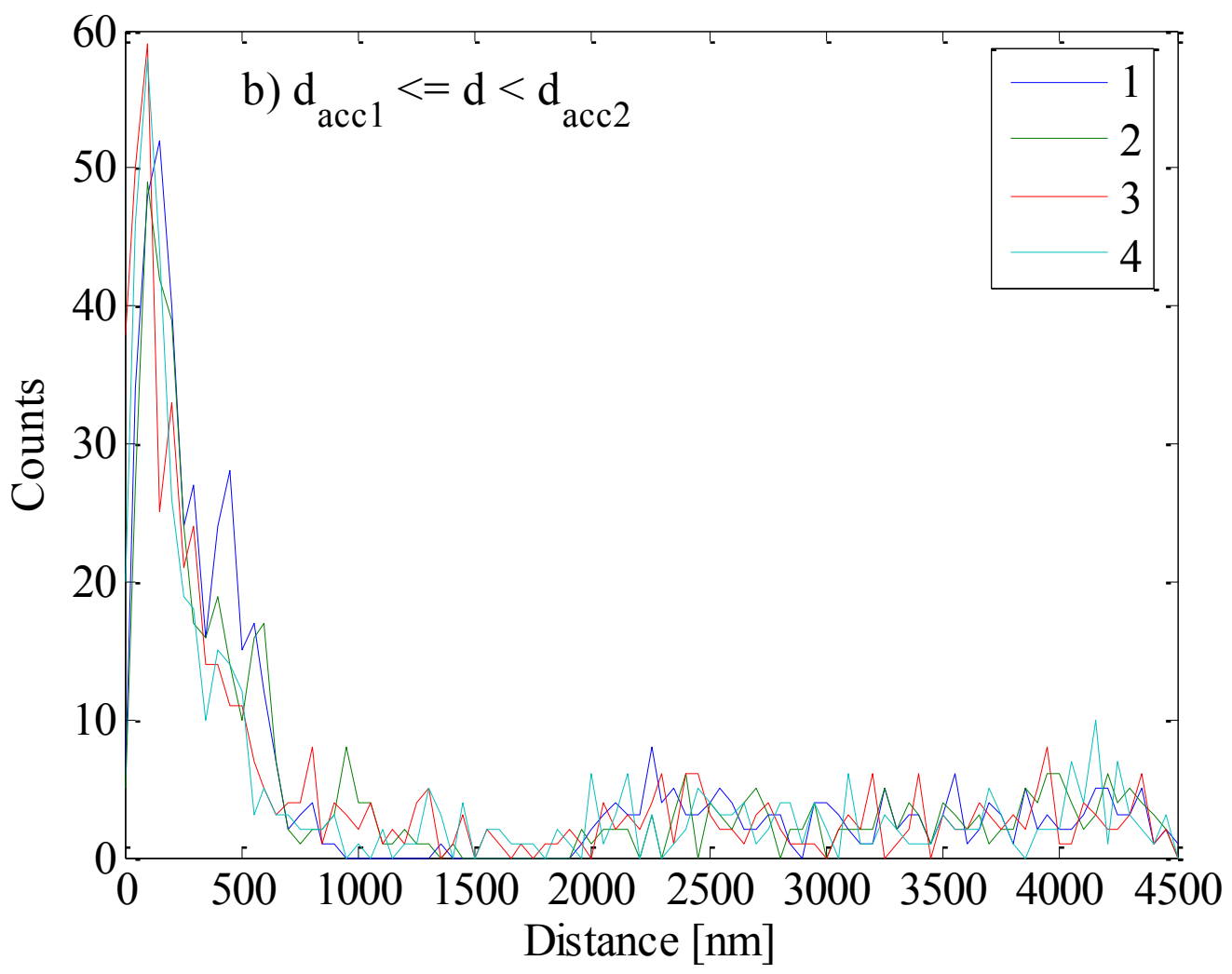




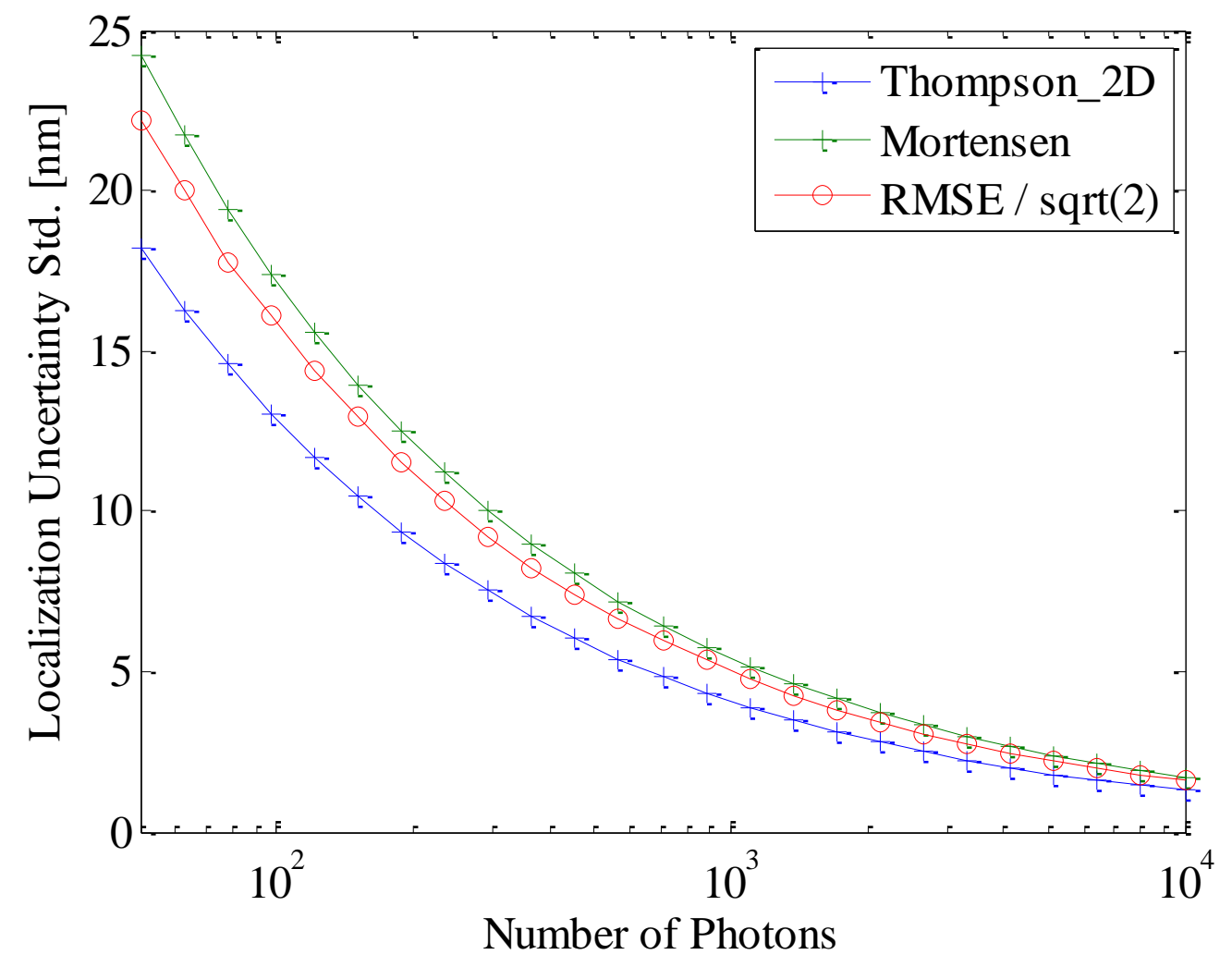


